\title{
Stiffness Control of Surgical Continuum Manipulators
}

\section{Citation}

Mahvash, M, and P E Dupont. 2011. "Stiffness Control of Surgical Continuum Manipulators." IEEE Transactions on Robotics 27 (2) (April): 334-345. doi:10.1109/tro.2011.2105410.

\section{Published Version}

doi:10.1109/TR0.2011.2105410

\section{Permanent link}

http://nrs.harvard.edu/urn-3:HUL.InstRepos:33884737

\section{Terms of Use}

This article was downloaded from Harvard University's DASH repository, and is made available under the terms and conditions applicable to Other Posted Material, as set forth at http:// nrs.harvard.edu/urn-3:HUL.InstRepos:dash.current.terms-of-use\#LAA

\section{Share Your Story}

The Harvard community has made this article openly available.

Please share how this access benefits you. Submit a story.

\section{Accessibility}




\title{
Stiffness Control of Surgical Continuum Manipulators
}

\author{
Mohsen Mahvash [Member, IEEE] and \\ Harvard Medical School, Brigham and Women's Hospital and the Cardiac Surgery Department, \\ Veterans Affairs Boston Healthcare System, West Roxbury, MA 02132 USA \\ Pierre E. Dupont [Fellow, IEEE] \\ Department of Cardiac Surgery, Children's Hospital Boston, Harvard Medical School, Boston, MA \\ 02115 USA \\ Mohsen Mahvash: mahvash@hms.harvard.edu; Pierre E. Dupont: pierre.dupont@childrens.harvard.edu
}

\begin{abstract}
This paper introduces the first stiffness controller for continuum robots. The control law is based on an accurate approximation of a continuum robot's coupled kinematic and static force model. To implement a desired tip stiffness, the controller drives the actuators to positions corresponding to a deflected robot configuration that produces the required tip force for the measured tip position. This approach provides several important advantages. First, it enables the use of robot deflection sensing as a means to both sense and control tip forces. Second, it enables stiffness control to be implemented by modification of existing continuum robot position controllers. The proposed controller is demonstrated experimentally in the context of a concentric tube robot. Results show that the stiffness controller achieves the desired stiffness in steady state, provides good dynamic performance, and exhibits stability during contact transitions.
\end{abstract}

\section{Index Terms}

Concentric tube robot; continuum robot; Cosserat rod; kinematics; stiffness control

\section{Introduction}

A continuum manipulator has the shape of a smooth curve, whose curvature can be controlled by adjusting the internal deformation of mechanically coupled elastic components of the body [1]. Continuum robots include steerable catheters [2], multibackbone snake-like robots [3], [4], and concentric tube robots [5], [6]. Two examples are shown in Fig. 1. Continuum manipulators are particularly useful for applications that require reaching inside confined spaces, such as is required in minimally invasive surgery. Thus, they have been proposed for use inside the heart [7], [8], the throat [4], and the sinuses [9].

Prior work on the control of continuum manipulators has been limited to position control [2], [3], [6]. For robotic tasks that involve interaction with an environment, stiffness control or its generalized form, i.e., impedance control, should be superior to position control. In impedance control, a robust dynamical relation between the environment force and the manipulator tip movement is established [10], [11].

\footnotetext{
() 2011 IEEE

This paper was recommended for publication by Associate Editor S. Hirai and Editor K. Lynch upon evaluation of the reviewers' comments.

Color versions of one or more of the figures in this paper are available online at http://ieeexplore.ieee.org.
} 
Furthermore, it can be useful to vary the stiffness of a continuum robot during minimally invasive surgery. For example, reduction of the stiffness of the robot's tip (or any other points on the robot) can enable safe navigation inside delicate confined spaces, e.g., to avoid wall puncture. In contrast, a high tip stiffness may be advantageous during tissue manipulation.

Stiffness control can also be used to provide haptic feedback during teleoperation of a continuum manipulator. In this scenario, master and slave controllers implement the same stiffness on the continuum manipulator and on the master arm held by the user [12], [13].

In general, the inputs of a stiffness controller are a tip-configuration set point and a desired stiffness. The desired tip force (or wrench) is calculated based on the difference between the set-point configuration and the actual configuration of the robot tip. For rigid robots, tip forces can be directly mapped to joint forces and torques using the transpose of the Jacobian matrix with the latter representing the differential mapping from joint positions to tip configuration. Thus, a rigid robot, by varying its joint torques, can theoretically vary its tip force with no displacement of its joints or tip. In practice, small joint displacements and link deflections do occur, but these are small enough to be negligible. Consequently, actual tip position can be calculated directly from joint positions.

In contrast, continuum robots are flexible by design, since robot shape is controlled by the storage and release of elastic energy in the robot's component parts. Thus, the kinematic mapping and the quasi-static force mapping are coupled. Configuration or force sensing at the robot's tip is needed to either directly measure tip configuration or to compute it. Furthermore, the mapping between actuator torques and tip forces in a continuum robot must include an additional term to account for the rate of elastic energy change [14].

The contribution of this paper is a stiffness controller that is easily applied to all types of continuum robots and does not require using force or torque sensors. The control law is developed in the context of a kinematic and static force map that is approximated by the product of two transformations: The first transformation calculates the unloaded kinematics of the robot, while the second calculates robot deformation due to applied loading. This approach has several advantages. First, it facilitates the use of robot deflection to both sense and apply tip forces. It also permits the use of efficient, noncontact kinematic models that are readily available for various types of continuum robots [2], [3], [5], [6].

The controller described here can be applied to any continuum robot that can be accurately approximated by a single Cosserat rod (of varying mechanical properties along its length) for the purpose of computing deformation due to external loading [15], [16]. This single-rod assumption applies when the relative motion between the elastic components of the robot in response to the application of external loads can be neglected. The rod model also neglects any shear of the robot's cross section or longitudinal extension due to applied loads and assumes linear constitutive behavior. These latter assumptions are accurate for long, thin continuum robots and are typical of those used in existing continuum robot kinematic models [2], [4], [5], [6], [14].

The Cosserat rod model enables computationally efficient calculation of robot deflection due to external forces by solving an initial value problem in which six state variables are integrated with respect to arc length [15], [17]. As demonstrated here, real-time implementation is straightforward, and furthermore, it has been shown that the error introduced by the rod approximation for concentric tube robots is small [17].

Gravity loading of the robot is not considered here, since the associated tip deflection is negligible $(<0.1 \mathrm{~mm})$ for the experimental robot considered here. If robot deflection due to 
gravity is important, it can be implemented with the controller by either of two means. The first approach is to incorporate it in the unloaded kinematic model of the robot. The second approach is to include it as a distributed load when computing the deflection using the rod model.

The paper is arranged as follows. Related work is described in the following section. Section III presents the general kinematic and force mappings of continuum robots. The deflection model using the special Cosserat rod model is presented in Section IV. In Section V, the stiffness controller is implemented using an iterative method to solve for the actuator positions that achieve the desired tip force. Efficacy of the proposed controller is demonstrated experimentally for a 3-degree-of-freedom (DOF) concentric tube robot in Section VI. Haptic feedback with matched stiffness controllers is also demonstrated. Conclusions appear in the final section.

\section{Related Work}

Unloaded kinematic models have been developed for a variety of continuum robots. Schematics of the two types appearing in Fig. 1 are shown in Fig. 2. Fig. 2(a) shows a concentric tube robot, which is constructed by telescopically extending concentrically combined precurved superelastic tubes. The robot's shape and tip location are controlled via the kinematic inputs consisting of the relative rotations and translations of the tubes at their proximal ends $\left(q_{1}, \ldots, q_{8}\right)$. The unloaded kinematic models for this type of robot depend on the modeling assumptions. When the tubes are considered torsionally rigid, the kinematics are described by algebraic equations [7]. For long or highly curved tubes, torsional compliance can be important and the kinematics take the form of differential equations in arc length with split boundary conditions [6].

Fig. 2(b) depicts a tendon-driven continuum robot of the type often used in steerable catheters and for distal dexterity enhancement in minimally invasive surgery. These robots possess a central flexible backbone that is deflected by symmetrically arranged wires [3], [18] or tubes [4], [14]. As shown in the schematic, spacer disks are attached to the central backbone. The wires or tubes slide through holes in all disks except for the most distal one to which they are attached. Unloaded kinematic models are developed by relating central backbone shape to tendon length $q_{1}, q_{2}$, and $q_{3}$ [3], [18]. While wire tendons are limited to tensile forces, tubes can also be used in compression and their bending stiffness must be accounted for in the kinematics [14], [19]. For this type of continuum robot, the mapping between forces in the actuating tubes and tip forces has also been derived [14]. Using this model, tube forces were used to infer tip forces.

Stability and vibration are of considerable concern when a flexible robot is in contact with its environment. This topic has been studied extensively in the literature in the context of both link flexibility using force control [20] and joint flexibility using impedance control [21]. A complete review of this literature and a stability analysis of the proposed controller are beyond the scope of this paper.

\section{Kinematic and Force Mappings of Continuum Robots}

All continuum robots can be modeled by a space curve $r(q, s) \in \Re^{3}$ that is a function of the $n$ kinematic input variables $q \in \mathfrak{R}^{n}$ and the arc length $s$, together with coordinate frames defined at the robot's base (frame $B$ ) and tip (frames $T$ land $T$ ). These are shown in Fig. 3. Referring back to Fig. 2, the space curve corresponds to the common tube centerline of (a) and the curve of the central backbone in (b). In contrast to rigid robots, two tip frames $T$ and $T$ are defined. The former corresponds to the tip configuration when no external loads are applied, while the latter includes the deformation arising from a wrench $F \in \mathfrak{R}^{6}$ applied 
externally at the tip. The first three components of $F$ correspond to the tip force, while the latter three are those of the torque applied to the tip [22]. All of the variables used in the remainder of the paper are defined in Table I.

The configuration of frame $C$ relative to frame $B$ is a rigid body transformation that can be written in homogeneous coordinates as follows:

$$
g_{b c}(q)=\left[\begin{array}{cc}
R_{b c}(q) & p_{b c}(q) \\
0 & 1
\end{array}\right]
$$

where $R_{b c} \in S O(3)$ is the rotation matrix, and $p_{b c} \in \mathfrak{R}^{3}$ is the translation vector between frames $B$ and $C$ [22]. This expression also represents a mapping from kinematic input space $q \in Q$ to the special Euclidean group $S E(3)$.

For the continuum robot of Fig. 3, the configuration of the frame $T$ relative to the frame $B$, $g_{b t}$, can be written as the product of two transformations

$$
g_{b t}(q, F)=g_{b \hat{t}}(q) g_{\widehat{t t}}(q, F)
$$

where $g_{b t}$ is the configuration of frame $T$ thelative to $B$, and $g_{t t}$ is the configuration of $T$ relative to $T !$ The first transformation $g_{b t}(q)$ corresponds to the unloaded robot kinematic models cited in Section II. The second transformation $g_{t t}(q, F)$ represents the displacement associated with the deformation of the robot due to the applied loading. Thus, the overall kinematic map $g_{b t}(q, F)$ is a function of both the kinematic variables and the external loading.

The force mapping equation for continuum robots can be derived from the principle of virtual work as was done in [14] to obtain

$$
\tau=J_{b t}^{T} F+\frac{\partial E(q, F)}{\partial q}
$$

Here, $T \in \Re^{n}$ is the vector of actuator forces and torques, $E(q, F) \in \Re$ is the elastic energy of the manipulator, and $J_{b t}(q, F)$ is the Jacobian matrix mapping actuator velocities to tip velocity [22]. Of course, for rigid robots, the elastic energy term drops out and this equation reduces to the standard form $\tau=J_{b t}^{T} F$.

To implement stiffness control in a rigid robot, the force mapping equation must be used to relate desired tip wrenches to actuator forces and torques. For continuum robots, however, it is also possible to implement stiffness control using the kinematic mapping (2). In this approach, (2) is solved for the actuator positions corresponding to the desired tip wrench $F^{d}$ and the actual measured tip configuration $g_{b t}^{m}$.

Comparing the two approaches, stiffness control based on the force mapping becomes an actuator force/torque control problem, while stiffness control using the kinematic map is an actuator position control problem. Use of the force mapping requires the use of joint force/ torque sensors [14] or actuator currents [12]. In contrast, actuator position control can be performed accurately using the existing actuator encoders.

Thus, it may be advantageous to employ (2) if it can be solved efficiently. To explore this question, assume that it is desired to apply a wrench $F_{d}$ at the tip of a 6-DOF continuum robot that has six independent joint variables, while its tip is held rigidly fixed at 
configuration $g_{b t}^{m}$. Equation (2) can be rewritten in terms of its inputs, measured tip configuration $g_{b t}^{m}$, and desired tip wrench $F^{d}$, and its output can be written in terms of its desired actuator positions $q^{d}$

$$
g_{b t}^{m}=g_{b \hat{t}}\left(q^{d}\right) g_{\widehat{t t}}\left(q^{d}, F^{d}\right) .
$$

Driving the actuator displacements to $q^{d}$ produces deformations of the continuum robot that produce the desired tip wrench $F_{d}$ at the measured tip configuration of the robot. Note that for the general case of contact with a soft environment, producing a desired tip wrench will also cause the tip configuration to change.

The root finding problem of solving (4) for $q^{d}$ can be performed efficiently if its right-hand side can be computed quickly. The term $g_{b t}(\cdot)$ is the unloaded forward kinematic model for which numerically efficient formulations are available [3], [6], [14], [18]. The second term, $g_{t t}(\cdot, \cdot)$ is the tip displacement produced by application of tip wrench on the unloaded robot.

As described in the following, given $g_{b t}\left(q^{d}\right)$, an efficiently computed estimate of the product $g_{b t}\left(q^{d}, F^{d}\right)=g_{b t}\left(q^{d}\right) g_{t t}\left(q^{d}, F^{d}\right)$ can be obtained using the special Cosserat rod model.

\section{Deflection Model}

To obtain $g_{b d}(q, F)=g_{b t}(q) g_{t}(q, F)$, we approximate any continuum manipulator as a single elastic rod, whose curvature and elastic properties match those of the robot for actuator values $q$ and tip wrench $F=0$. Thus, the curvature is selected to match the robot's backbone curve $r(q, s)$, and its stiffness is selected to match the composite stiffness of all elastic components that comprise the robot.

Deflection of the robot is computed as deflection of the rod in response to tip wrench $F$. This model is approximate in that it does not account for relative motions of the robot's elastic components in response to the tip wrench. As demonstrated in our experiments, the error associated with this approximation can be negligible. For more general cases, when the tip wrench causes significant relative motion between the elastic components of a continuum robot, the deflection of the robot can be calculated by modeling the robot with several Cosserat rods [17]. This general case is, however, beyond the scope of the paper.

The special Cosserat rod model is well known in the mechanics literature [16] and has also been employed in the robotics and computer graphics literatures [6], [15], [23]. Here, a concise overview of the model and its numerical solution are presented.

\section{A. Strain and Curvature of a Rod}

As shown in Fig. 3, coordinate frames can be defined along the backbone curve $r(q, s)$. In the following, actuator values $q$ are assumed constant and are omitted as arguments. Since these frames are intended to track material deformations of the rod's cross sections, a natural choice for frame orientation is to choose a base frame $B$ with one axis (i.e., the $z$-axis) aligned with the curve's tangent and then to slide this frame without rotation about the local $Z$-axis along $r(s)[24]$.

The resulting Bishop frame $P$ at arc length $s$ can be written as $g_{b p}(s), s \in[0, I]$, where $l$ is the length of the robot. Its origin lies on $I(s)$ and experiences a body-frame rate of change with respect to arc length $v(s) \in \mathfrak{R}^{3}$ given by 


$$
v(s)=R_{b p}^{-1}(s) \frac{d r(s)}{d s}
$$

where $R_{b p}(s)$ is the rotation matrix of $g_{b p}(s)$. The rate of change of frame $P$ s coordinate axis unit vectors $R_{b p}(s)=\left[e_{X}(s), e_{y}(s), e_{Z}(s)\right]$ with respect to arc length satisfy the bodyframe equations

$$
\frac{d R_{b p}}{d s}=R_{b p}(s)[u(s)]
$$

in which $u(s) \in \mathfrak{R}^{3}$ and the square bracket on the vector $u$ indicates the skew-symmetric matrix form

$$
[u(s)]=\left[\begin{array}{ccc}
0 & -u_{3} & u_{2} \\
u_{3} & 0 & -u_{1} \\
-u_{2} & u_{1} & 0
\end{array}\right]
$$

The vectors $u(s)$ and $v(s)$ are the angular and linear strains, respectively, experienced by the cross section. Thus, $u(s)$ has the units of curvature, and its $x$ - and $y$-components correspond to the bending of the rod, while its $z$-component corresponds to the twisting of the rod. Similarly, the $x$ - and $y$-components of $v$ are the shear-strain components of the cross section, while the $Z$-component is $V_{Z}=1+\epsilon_{Z}$ in which $\epsilon_{Z}$ is the longitudinal strain. Given the assumptions of negligible shear and longitudinal strain

$$
v(s)=\left[\begin{array}{lll}
0 & 0 & 1
\end{array}\right]^{T}, s \in[0, l]
$$

Angular and linear strains $u$ and $v$ provide body-frame descriptions of the curved shape of the rod. It can be helpful to note that $u$ and $v$ are analogous to body-frame angular and linear velocities if time is substituted for arc length. Thus, coordinate frames $g_{b p}(s)$ are obtained as the solution to the differential equation

$$
\frac{d}{d s}\left(g_{b p}(s)\right)=g_{b p}(s)\left[\begin{array}{cc}
{[u(s)]} & v \\
0 & 0
\end{array}\right] .
$$

This differential equation can be integrated numerically from base to tip or tip to base using a method that preserves the group structure of $S E(3)$. A variety of numerical integration methods are available for this purpose [25], [26].

The initial curvature of the robot prior to the application of external loads is denoted by $\hat{u}(s)$. Given an unloaded kinematic model that computes $g_{b p}(s)$, the unloaded shape of the rod used to model robot deflection can be computed as follows:

$$
[\widehat{u}(s)]=R_{b p}^{-1}(s) \frac{d R_{b p}(s)}{d s} .
$$

\section{B. Rod Deformation Due to Applied Loads}

To compute rod deformations, two equations are needed. The first is a constitutive model that relates cross-section strains $u$ and $v$ to the bending moment $m \in \Re^{3}$ and force $n \in \Re^{3}$ 
acting on the cross section. Since shear of the cross section and rod extension are neglected, only the relationship between $u$ and $m$ is needed. The second equation relates cross-section bending moment and force to the external loads. Both are described in the following.

When a rod with initial curvature $\hat{u}(s)$ is deformed to a new curvature $u(s)$, a bending moment $m(s)$ is generated. Assuming linear elastic behavior, the bending moment at any point $s$ along the rod is given by

$$
m(s)=K(s)(u(s)-\widehat{u}(s))
$$

where $K(s)$ is the frame-invariant stiffness tensor. For a large class of rods, $K(s)$ is given by

$$
\begin{gathered}
K(s)=\left[\begin{array}{ccc}
k_{x}(s) & 0 & 0 \\
0 & k_{y}(s) & 0 \\
0 & 0 & k_{z}(s)
\end{array}\right] \\
=\left[\begin{array}{ccc}
E_{x}(s) I_{x}(s) & 0 & 0 \\
0 & E_{y}(s) I_{y}(s) & 0 \\
0 & 0 & J_{p}(s) G(s)
\end{array}\right]
\end{gathered}
$$

$E_{X}(s)$ and $E_{y}(s)$ are moduli of elasticity, $I_{X}(s)$ and $I_{y}(s)$ are area moments of inertia, $J_{p}(s)$ is the polar moment of inertia, and $G(s)$ is the shear modulus. These values should be selected so that the rod stiffness matches the overall continuum robot stiffness as a function of arc length.

To relate cross-section bending moment and force to the external loads, we employ the equilibrium equation of the special Cosserat rod model [16]. Written in the body-frame coordinates of Fig. 3, the differential equation governing bending moment $m$ and force $n$ as a function of arc length $s$ is given by

$$
\begin{gathered}
\frac{d m(s)}{d s}=\eta(s)-[u(s)] m(s)-[v(s)] n(s) \\
\frac{d n(s)}{d s}=\varphi(s)-[u(s)] n(s)
\end{gathered}
$$

where $\varphi(s)$ and $\eta(s)$ are the applied force and torque per unit length of the rod and the bracket notation is as defined in (7).

For simplicity of exposition, it is assumed that only tip loads are applied to the robot, and therefore, $\eta(s)=\varphi(s)=0$, and $s \in[0, I]$. Combining (11) and (13) results in equations for curvature $u$ and force $n$

$$
\begin{gathered}
\frac{d u(s)}{d s}=\frac{d \widehat{u}(s)}{d s}-K^{-1}(s)\left([u(s)] K(s)(u(s)-\widehat{u}(s))+[v] n(s)+\frac{d K(s)}{d s}(u(s)-\widehat{u}(s))\right) \\
\frac{d n(s)}{d s}=-[u(s)] n(s) .
\end{gathered}
$$

The boundary conditions for these equations $u(I), n(I)$ can be specified from the body-frame wrench $F_{b}$ applied at the robot's tip

$$
\begin{aligned}
& {\left[n^{T}(l) m^{T}(l)\right]^{T}=F_{b}} \\
& u(l)=K^{-1} m(l)+\widehat{u}(l)
\end{aligned}
$$


and (14) can be solved numerically by integrating backward in arc length from $s=1 \rightarrow 0$ [15]. Since (13) is written in body coordinates, the body-frame twist velocity $\left[v^{T}, u^{T}\right]^{T}$ must be simultaneously integrated such that the equations evolve on $S E(3)$. As detailed in [15], a first-order Crouch-Grossman method [25] provides computational efficiency. The result of this integration is the desired estimate of the transformation between the base and tip of the robot $g_{b t}=g_{b t} \rrbracket g_{t t}$.

Note that in the case of a multisection continuum robot, there are often discontinuities in $K(s)$ and $\hat{u}(s)$ at the section boundaries. In these cases, integration of (14) is performed section by section from the tip to the base. While (15) provides the initial value for $u(s)$ at the tip of the robot, the initial value for each subsequent section is obtained by equating bending moments across section boundaries and using (11) to solve for the corresponding initial value of $u(s)$ to use in (14).

In stiffness control, the desired tip wrench $F_{w}^{d}$ is often calculated in a local frame at the tip of the robot, whose axes always remain parallel to the axes of the world frame.

In this case, the boundary condition is a function of the desired shape of the rod.

Specifically, if the base of the rod is positioned at the origin of the world frame, then $g_{W} b$ in

Fig. 3 is the identity matrix and the desired body-frame tip wrench $F_{b}^{d}$ to be used in (15) is related to the desired world frame tip wrench by

$$
F_{b}^{d}=\left[\begin{array}{cc}
R_{b t}^{T} & 0 \\
0 & R_{b t}^{T}
\end{array}\right] F_{w}^{d}
$$

where $R_{b t}$ is the rotation matrix of $g_{b t}\left(q^{d}, F_{b}^{d}\right)$. While the integral must now be solved iteratively, convergence is rapid when the tip wrench varies smoothly and the solution is seeded using the desired rod shape from the previous time step.

\section{Stiffness Controller}

The task of the controller is to create a user-defined stiffness at the tip of the continuum manipulator. If the stiffness is specified for all DOF of the robot tip, a pure stiffness controller can be implemented. In many medical applications, however, it is desirable to control the stiffness in certain directions, e.g., tip position, and to control the motion in other directions, e.g., tip orientation. Because of its practical importance, this latter case of hybrid stiffness/motion control is considered here. The framework described in the following can be easily adapted to other combinations of stiffness- and motion-controlled coordinates.

The desired tip force $f^{d} \in \mathfrak{R}^{3}$ is computed using the user-defined diagonal stiffness matrix $K^{d} \in \mathfrak{R}^{3 \times 3}$, based on the difference between measured robot tip position $p_{b t}^{m} \in \mathfrak{R}^{3}$, and a reference tip position $p_{b t}^{r} \in \mathfrak{R}^{3}$ as follows:

$$
f^{d}=K^{d}\left(p_{b t}^{m}-p_{b t}^{r}\right)
$$

The desired tip orientation of the robot is given as $R_{b t}^{d} \in S O$ (3).

As shown in Fig. 4, the stiffness equation (17) can be pictured as a virtual spring of stiffness $K^{d}$ that connects the reference position $p_{b t}^{r}$ to the current tip position of the manipulator. 
The desired force $f^{d}$ and desired tip orientation $R_{b t}^{d}$ must be mapped to the actuator positions $q^{d}$, such that the deflected robot will generate the force $f^{d}$ at the tip configuration

$$
g_{b t}^{d m}=\left[\begin{array}{cc}
R_{b t}^{d} & p_{b t}^{m} \\
0 & 1
\end{array}\right]
$$

The desired actuator positions are implicitly defined by (4), which is rewritten for $g_{b t}^{d m}$

$$
g_{b t}^{d m}=\left[\begin{array}{cc}
R_{b t}^{d} & p_{b t}^{m} \\
0 & 1
\end{array}\right]=g_{b t}\left(q^{d}, F_{w}^{d}\right)=g_{b \hat{t}}\left(q^{d}\right) g_{\widehat{t t}}\left(q^{d}, F^{d}\right) .
$$

In this equation, $p_{b t}^{m}$ is directly measured by a tip sensor, while $g_{b t}\left(q^{d}, F_{w}^{d}\right)$ is computed for values of $q^{d}$ and $f^{d}$ from the rod deformation model (14)-(16) using the wrench boundary condition

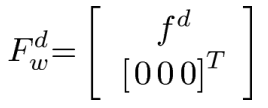

and the initial shape (10) obtained from the unloaded kinematic model,

$$
\left[\widehat{u}\left(q^{d}, s\right)\right]=R_{b p}^{-1}\left(q^{d}, s\right) \frac{d R_{b p}\left(q^{d}, s\right)}{d s}
$$

in which $R_{b p}\left(q^{d}, s\right)$ is the rotation matrix of $g_{b p}\left(q^{d}, s\right)$. Note that (20) assumes that the torque applied to the robot by the environment is zero. In situations involving large contact torques, tip torque sensing is necessary and measured torque values should be used in (20).

Using an efficient numerical implementation, e.g., Gauss-Newton, (19) can be solved iteratively at each time step of the controller for the actuator positions $q^{d}$ that produce the desired combination of tip force and orientation. By driving the actuator variables to $q^{d}$, the manipulator is deformed to produce the desired tip force $f_{d}$ and desired orientation $R_{b t}^{d}$.

Position-tracking controllers, e.g., PD, can be used to drive and maintain the actuators of the manipulator at their desired values. The gains of the actuator controllers should be selected to achieve the desired stiffness controller bandwidth and such that the steady-state tip position error due to actuator error is small, compared with the deflection of the manipulator.

\section{A. Stiffness Control Using a Modified Position Controller}

Since position controllers already exist for a variety of continuum robots [2], [3], [6], it can be advantageous to leverage the existing controller implementation to achieve stiffness control. This can be accomplished as follows.

A position controller solves the inverse kinematic problem and drives the actuators to the positions given by 


$$
q^{d}=\mathscr{I}\left(g_{b \widehat{t}}^{d}\right)
$$

where $\mathscr{f}(\cdot)$ represents a multidimensional inverse kinematic function for the unloaded robot that calculates either numerically or analytically the actuator positions corresponding to the user-defined configuration $g_{b \widehat{t}}^{d}$.

To include the tip-applied wrench, the argument of $\mathscr{f}$ can be rewritten using (19) to obtain

$$
q^{d}=\mathscr{I}\left(g_{b t}^{d m} g_{\widehat{t t}}^{-1}\left(q^{d}, F_{w}^{d}\right)\right)
$$

where the Cosserat deflection model is used to compute $g_{\widehat{t t}}\left(\cdot, F_{w}^{d}\right)$.

This equation can be solved for $q^{d}$ using fixed-point iteration [27]

$$
q_{i}^{d}=\mathscr{I}\left(g_{b t}^{d m} g_{\widehat{t t}}^{-1}\left(q_{i-1}^{d}, F_{w}^{d}\right)\right), i=1,2, \ldots
$$

The convergence condition for (24) is obtained using the fixed-point iteration condition of [27]

$$
|| J_{\mathscr{I}\left(g_{b t}^{d m}\right.} \underset{\left.g_{t t}^{-1}\left(q, F_{w}^{d}\right)\right)}{ }||_{\infty}<1
$$

where $J \mathscr{f}=\partial \mathscr{I} / \partial q$ is the Jacobian of $\mathscr{I}$ with respect to actuator positions $q$. For any given continuum robot, this condition can be evaluated numerically or analytically to ensure convergence.

This way, the stiffness controller can be implemented by the online iteration of (24). The controller computations for each iteration consist of a single evaluation of 1) $g_{\widehat{t t}}\left(q_{i}^{d}, F_{w}^{d}\right)$ and 2 ) the unloaded inverse kinematic model $\mathscr{I}(\cdot)$. Note that the deflection model is calculated from $g_{\widehat{t t}}\left(q_{i}^{d}, F_{w}^{d}\right)=g_{b \widehat{t}}^{-1}\left(q^{d}\right) g_{b t}\left(q^{d}, F_{w}^{d}\right)$.

In general, the iteration equation (24) can be initialized with the measured actuator positions of the robot or the desired actuator positions from the previous cycle of the controller and should be evaluated until convergence at each cycle of the controller. It is often sufficient, however, to evaluate the iteration equation (24) once per cycle using the desired actuator positions from the previous cycle of the controller. This is possible because most preexisting position controllers run at a much higher rate (e.g., $1000 \mathrm{~Hz}$ ) than the bandwidth of the manually commanded controller inputs $p_{b t}^{r}$ and $R_{b t}^{d}$ (e.g., $<10 \mathrm{~Hz}$ ). One iteration will be enough to keep the desired actuator positions close to the iteration solution. Note that even for a step change in input, (24) still converges when (25) is satisfied.

Fig. 5 shows the block diagram of the stiffness controller when a single iteration is employed at each time step. The controller calculates the stiffness force using the measured tip position and the reference tip position generated by the master arm. The stiffness force is then used to calculate the desired deflection and, from it, the desired undeformed configuration of the robot tip. This comprises the input to the position controller of the 
robot, which generates the actuator torques. As described in the next section, a feedforward term is added to actuator torques to achieve stiffness control along the axis of the robot.

\section{B. Stiffness Control Along the Axis of the Robot}

Since most continuum robots possess a high axial stiffness, deformation due to tip loading was modeled using an inextensible Cosserat rod model. When the robot configuration is such that its curvature is close to zero along its entire length, the stiffness in the tip tangent direction approaches the high axial stiffness value.

In the neighborhood of this straight configuration, the stiffness controller, which is implemented using (24), will not produce the desired force component in the axial direction. To see this, consider the case when the desired wrench consists solely of a force in the axial direction. In this case, no deformation is predicted by the inextensible model, and therefore, $g_{b t}\left(q_{d}, F_{w}^{d}\right)=g_{b \widehat{t}}\left(q_{d}\right)$. Substituting this result in (24) shows that the stiffness controller will maintain the robot at its current position $p_{b t}^{m}$.

In this direction, however, the energy term in the force mapping equation of (3) goes to zero, and the map from tip force to actuator force along this axis reduces to the standard Jacobian transpose. Thus, actuator current can be used in the conventional way to control tip force and stiffness in this direction. The standard conditions for using this approach also apply here. The actuator transmission must be backdrivable to ensure that the force produced at the tip is the same as the force applied by the actuator. Friction compensation may also be necessary in order to minimize error in the force produced at the tip.

To enable stiffness control in these configurations, the controller can be modified as follows. For simplicity of exposition, it is assumed that a single actuator $j$ controls displacement of the robot along the $z$-axis at its base. It is assumed that the joint controller for actuator $j$ is a proportional controller augmented with friction compensation. To modify the controller, the component of desired tip force along $z, f_{z}^{d}$, is added to the actuator force, as shown in the block diagram of Fig. 5, and this results in

$$
f_{j}^{a}=f_{z}^{d}+f_{f}+k_{j}^{p}\left(q_{j}-q_{j}^{d}\right)
$$

in which $f_{j}^{a}$ is the actuator force, $f_{f}$ is the friction compensator force, $k_{j}^{p}$ is the proportional gain of the position controller, and $q_{j}$ and $q_{j}^{d}$ are the current and desired positions of actuator $j$, respectively. A gravity compensation term can also be included as needed. Note that, as feedforward terms that act to cancel output error, they can be applied whether or not the robot is straight, since they always act to reduce actuator error.

\section{Experimental Implementation}

The stiffness controller was implemented to produce a desired positional tip stiffness on a 3DOF concentric tube robot. This is a case of pure stiffness control, since the stiffness is specified for each DOF. The robot was comprised of the two 150-mm-long tubes shown unassembled in Fig. 6 and assembled in Fig. 7. Relative rotation of the tubes varies their combined curvature $u(s)$ from the initial constant precurvature value of $u(s)=\left[\begin{array}{llll}1 / 240 & 0 & 0\end{array}\right]^{T}$ $\mathrm{mm}^{-1}, s \in[0,150] \mathrm{mm}$, when the curvatures are aligned $\left(q_{1}=q_{2}\right)$ to $u(s)=\left[\begin{array}{lll}0 & 0 & 0\end{array}\right]^{T}, s \in[0$, $150] \mathrm{mm}$ (robot is straight) when the curvatures are antialigned $\left(q_{1}=q_{2}+\pi\right)$. The robot can also be translated in the $z$-direction by actuator displacement $q_{3}$. Varying $\left\{q_{1}, q_{2}\right\} \in S O(2) \times$ $S O(2)$ and $q_{3} \in R$ produces a cylindrical workspace at the robot's tip of radius $48 \mathrm{~mm}$ and length $254 \mathrm{~mm}$. 
An existing position control architecture [6] was modified to implement stiffness control. The existing controller consists of a master-slave system in which the concentric tube manipulator is the slave arm, and a PHANTOM Omni haptic device (Sensable Technologies, Inc.) is employed as the master arm. The position controller is implemented as a multithreaded process under Windows 2000. The process includes two time-critical user-mode threads running at $1 \mathrm{kHz}$ that implement the kinematic model and PD joint controllers and an application thread that updates a GUI.

The stiffness controller requires real-time measurement of the robot's tip configuration. This was accomplished using an electromagnetic-tracking sensor (3-D Guidance trakSTAR, Ascension Technology Corporation). The $2 \times 9.7 \mathrm{~mm}$ cylindrical sensor (model 180) was attached to the robot's tip, as shown in Fig. 6 . The sensor includes a small cylindrical probe of $9.7 \mathrm{~mm}$ length and $2 \mathrm{~mm}$ radius and a white cable (visible in the figures) that connects the probe to its electronics. Sensor accuracy is $1.4 \mathrm{~mm}$ RMS in translation and $0.5^{\circ} \mathrm{RMS}$ in rotation with a resolution of $0.5 \mathrm{~mm}$ and $0.1^{\circ}$. The update rate of the sensor was set to 100 Hz. The sensor's electrical leads produced negligible deformation of the robot.

To calibrate the deflection model used in the stiffness controller and to evaluate the controller's performance, a 22-N tension/compression load cell (Sensotec model 31) was used to measure environment force. The load cell was connected to the tip of the manipulator through a long thin cord to prevent the metal components of the load cell from distorting the magnetic field of the tip-tracking system. This loading configuration is depicted in Figs. 8 and 9. Note that the force measurements are not used by the controller.

To evaluate the controller, experiments were performed with both moving and fixed environment models. A moving environment was produced, as shown in Fig. 8, by manually pulling on the robot tip in a desired direction through a cord attached to a load cell. During these tests, the desired reference tip position $p_{b t}^{r}$ of (17) was held constant by fixing the position of the master.

A soft stationary environment was produced, as shown in Fig. 9, by attaching the robot tip to a fixed load cell through a rubber band. In these tests, the master arm was used to move the desired reference tip position $p_{b t}^{r}$ of (17) in the desired direction.

Implementation of the proposed stiffness controller requires an unloaded kinematic model and a calibrated deflection model. Each is described next, followed by the results of the control experiments.

\section{A. Unloaded Kinematic Model}

To implement stiffness control by modification of a position controller as given by (24), it is assumed that forward and inverse kinematic solutions are already implemented for the noncontact case. Such models have been presented in [6] for concentric tube continuum robots. Modified versions of these models, which are appropriate to the pair of tubes used in the experiments, are presented here.

While, in general, the combined curvature of two tubes of constant precurvature varies along their length due to torsional twisting of the tubes [6], this effect is negligible for the tubes used in the experiments. Thus, it is appropriate to model the combined curvature as a function of actuator values $\left\{q_{1}, q_{2}\right\}$ that is independent of arc length. It can be written in the world frame of Fig. 7 as follows: 


$$
u^{w}=A_{\kappa}\left(q_{1}-q_{2}\right)\left[\begin{array}{c}
\cos \left(\left(q_{1}+q_{2}\right) / 2+\varphi_{\kappa}\left(q_{1}-q_{2}\right)\right) \\
\sin \left(\left(q_{1}+q_{2}\right) / 2+\varphi_{\kappa}\left(q_{1}-q_{2}\right)\right) \\
0
\end{array}\right]
$$

Here, $A_{K}(\cdot)$ and $\varphi_{K}(\cdot)$ compute the magnitude and phase of curvature as functions of the relative tube rotation angle $q_{1}-q_{2}$. For curve fitting, $A_{K}(\cdot)$ and $\varphi_{K}(\cdot)$ are interpreted as the magnitude and phase of a complex function $\kappa(\cdot)$.

The tip position, assuming no contact forces, is obtained from the curvature $u^{W}$ as follows $[7]$ :

$$
p_{b \hat{t}}(q)=\left[\begin{array}{c}
\frac{u_{y}^{w}\left(1-\cos \left(l\left|u^{w}\right|\right)\right.}{\left|u^{w}\right|^{2}} \\
\frac{-u_{x}^{w}\left(1-\cos \left(l\left|u^{w}\right|\right)\right.}{\left|u^{w}\right|^{2}} \\
q_{3}+\frac{\sin \left(l\left|u^{w}\right|\right)}{\left|u^{w}\right|}
\end{array}\right]
$$

in which $l$ is the arc length of the manipulator. To obtain the most accurate kinematic model, the complex function $K(\cdot)$ was calculated from (27) and (28) as a truncated Fourier series using position measurements obtained with the tip-tracking sensor over two complete revolutions of the tubes.

\section{B. Deflection Model Calibration}

To calculate robot deflection due to tip loading, the deflection model requires the unloaded body-frame curvature of the robot $\hat{u}(s)$, as well its composite stiffness $K(s)$. While (10) provides a general expression for unloaded curvature, in this case, it can be directly obtained from (27). Due to the choice of Bishop body frames and since the unloaded kinematic model is of constant curvature

$$
\widehat{u}(s)=u^{w} .
$$

The deflection model approximates the composite stiffness of all elastic elements of the robot by the matrix of bending and torsional stiffnesses $K(s)$ defined in (12). Since the robot is composed of NiTi tubes, $K(s)$ reduces to

$$
K(s)=K=\operatorname{diag}\left(E_{c} I_{c}, E_{c} I_{c}, E_{c} I_{c} /(1+\nu)\right)
$$

in which $E_{\mathcal{c}}$ and $I_{\mathcal{c}}$ are the composite values for elastic modulus and area moment of inertia, respectively, and $v$ is Poisson's ratio.

Using the value of $v=0.3$ that is appropriate for $\mathrm{NiTi}, E_{c} I_{c}$ was estimated experimentally using the testing configuration of Fig. 8 and the maximum possible value of initial curvature $\hat{u}(s)=\left[\begin{array}{lll}1 / 240 & 0 & 0\end{array}\right]^{T} \mathrm{~mm}^{-1}$. An iterative method was used to solve for the stiffness matrix that minimized the error between the force-displacement response predicted by the model and that obtained by measurement in $x$-and $y$-directions. The resulting calibrated stiffness of

$$
K(s)=\operatorname{diag}\left(\begin{array}{lll}
0.049 & 0.049 & 0.038
\end{array}\right) N \cdot m^{2}
$$


was used to compare the deflection model and experimental robot tip stiffnesses, as shown in Fig. 10. (See Fig. 7 for the coordinate directions.) The depicted experimental data was collected for cyclic displacements in the $x$-, $y$-, and $z$-coordinate directions, while holding the robot actuators fixed. While the experimental data reveals a small amount of hysteresis, the deflection model provides a good fit with the loop average in the $x$-and $y$-directions, while the high stiffness in the $z$-direction reduces the accuracy of the model fit. Detailed analytical and experimental evaluations of the model can be found in [17].

Fig. 10 can also be used to understand how the robot tip stiffness varies with curvature. As the curvature is reduced from its maximum $\hat{u}(s)=\left[\begin{array}{llll}1 / 240 & 0 & 0\end{array}\right]^{T} \mathrm{~mm}^{-1}$ to its minimum $\hat{u}(s)=$ $\left[\begin{array}{lll}0 & 0 & 0\end{array}\right]^{T} \mathrm{~mm}^{-1}$, the stiffness in the $x$-direction remains almost constant, while the $y$ - and $z$ direction stiffnesses change smoothly. (Note that $\{x, y, z\}$ directions are the directions of the world frame, as shown in Fig. 7 and, therefore, do not change during the tests.) The $y$ direction stiffness converges to that of the $x$-direction. This is expected since a straight rod aligned with the $z$-axis should have equal tip stiffnesses in the $x$ - and $y$-directions. The $z$ direction stiffness increases, as depicted in Fig. 10, toward an infinite value as the curvature approaches zero. This matches the inextensibility assumption of the rod model.

\section{Stiffness Controller}

Stiffness control was implemented for the 3 DOF continuum robot depicted in Fig. 7 by modifying an existing position controller. The position controller uses Newton's method to solve (27) and (28) at each time step for the actuator positions associated with the desired unloaded tip position $p_{b \widehat{t}}^{d}$

$$
q^{d}=\mathscr{I}\left(p_{b \widehat{t}}^{d}\right)
$$

PD controllers are used to drive the actuators to the values computed in (32). In addition, friction compensation and stiffness force $f_{z}^{d}$ are implemented on the linear axis $q_{3}$, as described in Section V-B and (26) to account for the high axial stiffness of the robot. Note that the mechanical transmission of $q_{3}$ is a non-backdrivable ball screw and that the effect of the feedforward force term was limited by inexact friction compensation.

To achieve stiffness control, (32) was replaced with the iteration equation (24), which, for this robot, reduces to an expression involving only tip positions

$$
q_{i}^{d}=\mathscr{I}\left(p_{b t}^{m}-\left(p_{b t}\left(q_{i-1}^{d}, F_{w}^{d}\right)-p_{b \widehat{t}}\left(q_{i-1}^{d}\right)\right)\right)
$$

Here, $p_{b t}^{m}$ is the current tip position as measured by the tip-tracking sensor. The unloaded tip position $p_{b \hat{t}}\left(q_{i-1}^{d}\right)$ is computed using (27) and (28).

The deflected tip position $p_{b t}\left(q_{i-1}^{d}, F_{w}^{d}\right)$ is calculated from the deflection model (14) using $q_{i-1}^{d}$ to compute the predeflected curvature $\hat{u}(s)$ from (27) and (29). The boundary conditions (15) are computed using the desired tip wrench $F^{W}$ as defined by (17) and (20). This wrench is converted to body coordinates using (16) with $R_{b t}$ computed using the current tip orientation as measured by the tip sensor. The integration was carried out with the discrete (in arc length) formulation detailed in [15] using ten nodes along the $150 \mathrm{~mm}$ length of the robot. 


\section{Controller Evaluation}

To evaluate the controller, four sets of experiments were conducted. The first two sets were conducted at low velocities in order to verify the quasi-static-tracking capabilities of the controller. These tests considered separately the controller performance for a moving environment and for a varying tip reference position. To characterize the dynamic performance of the robot, the third and fourth sets of tests address two important cases of environment interaction. In the third set of tests, a step change in tip reference position is applied to evaluate the transient response when moving from free space to environment contact. The fourth set of tests evaluate the effect of tip reference position frequency on controller stiffness during sustained contact. Each set of tests is described in the following section.

1) Fixed Robot Reference Position With Moving Environment-The testing configuration of Fig. 8 was used to evaluate the performance of the controller in the three coordinate directions for various values of robot curvature and tip stiffness. During these tests, the master manipulator was held fixed such that the reference tip position $p_{b t}^{r}$ was constant and lay in the $y$-z-plane above the line defined by actuator axis $q_{3}$ (see Fig. 7). In each test, the robot tip was displaced in one of the three coordinate directions. Each displacement started with the robot tip in the unloaded configuration and proceeded until an arbitrary maximum value was obtained. The displacement was then reversed.

Fig. 11 depicts the measured tip force and displacement in the three coordinate directions of Fig. 7 for an intermediate value of noncontact robot curvature given by $u^{W}=[(1 / 320 \mathrm{~mm}) 0$ $0]^{T}$. As is also shown, the desired stiffnesses of (17) were set to be equal in the three coordinate directions $K_{d}=\operatorname{diag}(0.040 .040 .04) \mathrm{N} / \mathrm{mm}$.

It can be seen that the desired stiffness is accurately achieved in the $x$ - and $y$-directions. Stiffness in the $z$-direction is less accurate, especially at direction reversals, where imperfect cancellation of friction in the ball screw transmission of actuator $q_{3}$ leads to a large amount of hysteresis. Furthermore, since force application in $z$-direction reduces the robot's curvature, the amplitude of displacement was limited to about $6 \mathrm{~mm}$ to ensure that the robot possesses some nonzero curvature over the entire trial. It is interesting to note, however, that the stiffness controller was successful in achieving a desired stiffness along the $z$-axis.

The most difficult configuration for stiffness control corresponds to when the robot is straight, i.e., the noncontact curvature is $u^{W}=\left[\begin{array}{lll}0 & 0 & 0\end{array}\right]^{T}$. Tip force versus displacement data for this configuration are shown in Fig. 12 for a desired stiffness of $K_{d}=\operatorname{diag}(0.020 .080 .2)$ $\mathrm{N} / \mathrm{mm}$. Recall that the natural stiffness in the $x$ - and $y$-directions for the straight robot should be both equal to about $0.048 \mathrm{~N} / \mathrm{mm}$, as depicted in Fig. 10. The stiffness controller has succeeded in reducing the natural robot stiffness by about a factor of two in the $y$-direction and in increasing the natural stiffness by about a factor of two in the $x$-direction. The stiffness controller (not depicted) was unable to change the natural stiffness of the robot in the $z$-direction due to inexact friction cancellation in the screw transmission.

2) Moving Robot Reference Position With Fixed Environment-The testing configuration of Fig. 9 was used to evaluate the performance of the stiffness controller in the $y$-coordinate direction when pushing (actually pulling) against a soft immobile object. During these tests, the master manipulator was positioned such that the reference tip position $p_{b t}^{r}$ was in the $y-z$-plane above the line defined by actuator axis $q_{3}$ (see Fig. 7). In each test, the robot tip started in the unloaded configuration. The master was moved in order to move $p_{b t}^{r}$ in the positive $y$-direction to an arbitrary maximum value. The displacement was then reversed. 
Fig. 13 plots the measured force versus $y$-direction displacement of the manipulator tip for two trials with the same environment for the two trials are 0.02 and $0.06 \mathrm{~N} / \mathrm{mm}$. The plots show that the desired stiffness is achieved with high accuracy but that the actual stiffness decreases somewhat for tip forces above $0.25 \mathrm{~N}$.

3) Contact Transition Due to Step Change in Tip Reference Position-These tests employed a modification of the testing configuration shown in Fig. 9 in which the rubber band was removed and the robot tip was connected directly by the cord to the environment load cell. During the tests, the reference tip position $p_{b t}^{r}$ was in the $y$ - $z$ - plane and was directly commanded along the $y$-axis by the computer without using the master arm. The reported results correspond to the dynamic performance of the stiffness controller in the $y$-coordinate direction.

In these tests, cord length was adjusted (see Fig. 9) such that the environment force is nonzero for $y>0$. The tip reference position is initially commanded to $y^{r}=-2 \mathrm{~mm}$ such that the cord is loose and that no force is applied to the robot. A step change to $y^{r}=8 \mathrm{~mm}$ is then commanded, and the robot moves upward so that the cord becomes and remains taut. This test evaluates stiffness control during contact transitions.

An example response for a desired stiffness $k_{d}=0.08 \mathrm{~N} / \mathrm{mm}$ is depicted in Fig. 14. As can be seen from Fig. 14(a), prior to the step input, the tip position is regulated to the reference position. Once the step is applied, the robot tip moves in free space for $2 \mathrm{~mm}$ before the cord becomes taut, and a force is generated. The measured tip force and position reach to their steady-state values almost $0.3 \mathrm{~s}$ after the step input.

The corresponding plot of force versus virtual spring displacement is plotted in Fig. 14(b). All time prior to application of the step maps to the origin of the plot. The moment of step application corresponds to the horizontal segment at zero force (and thus, zero stiffness). The subsequent transient response is observed to spiral in and converge to a point on the commanded stiffness line. These results demonstrate the stability of the stiffness controller and, based on a settling time of $0.3 \mathrm{~s}$, suggest a bandwidth of several hertz. To take a closer look at controller bandwidth during environment contact, the following tests were performed.

4) Effect of Reference Position Frequency on Stiffness Control-In these tests, the tip reference position, $y^{r}$ was commanded to move sinusoidally. The testing configuration was similar to the step input tests described earlier, except that the cord length was now adjusted such that it was in tension for the entire range of commanded reference positions. Data was collected for sinusoidal inputs $y^{r}$ of frequencies $f_{i}=0.5,1,2,3,4$ and amplitude $A=12 \mathrm{~mm}$

$$
y^{r}(t)=A \sin \left(2 \pi f_{i} t\right)
$$

Tip force versus virtual spring displacement for a desired stiffness of $k_{d}=0.02 \mathrm{~N} / \mathrm{mm}$ is shown in Fig. 15. The plot reveals that the width of force-displacement loop increases with the frequency of the input $y^{r}$. The main reason for this dependency is the limited bandwidth of the joint controllers in the experimental system $(\approx 5 \mathrm{~Hz})$.

In additional teleoperation experiments, haptic feedback was implemented by setting the desired tip stiffnesses of both the master and continuum-robot slave to $K_{d}=\operatorname{diag}(0.040 .04$ $0.04) \mathrm{N} / \mathrm{mm}$. This control scheme is similar to a position-position architecture and provides 
force transparency but does not provide stiffness transparency when the environment is stiffer than the controller [28].

Further tests also showed that the stiffness controller starts becoming unstable when the desired stiffness values of the controller approach twice the inherent stiffness values of the manipulator in each direction [21]. The tests were performed with both soft and rigid environments.

\section{Conclusion}

Continuum robots are a relatively novel class of manipulators that is increasingly being adapted to medical applications. Since these robots achieve their shapes through deformation of elastic members along their length, kinematic modeling, and real-time control of these robots are substantially more difficult than for traditional robots with rigid links and discrete joints. Consequently, control of these robots has, to date, been limited to position control. In medical applications, however, it is important to control the interaction forces with soft and delicate tissue.

The contribution of this paper is to provide an approach for implementing stiffness control on any continuum robot that can be modeled under loading as an elastic rod and for which an unloaded kinematic model is available. Thus, the method is broadly applicable to continuum robots including steerable catheters, multibackbone robots, as well as concentric tube robots.

The efficacy of the proposed stiffness controller was demonstrated on a 3-DOF concentric tube robot. It was found that desired tip stiffnesses could be achieved independent of robot configuration in the lateral or bending directions. Along the axis of the robot, stiffness control can be implemented using the standard Jacobian transpose force mapping as long as the robot is backdrivable.

While controller stability was not addressed analytically, experiments showed the controller to be stable for desired stiffnesses less than twice the natural stiffness of the robot. Future work will formally address the stability of the controller.

\section{Acknowledgments}

This work was supported by the National Institutes of Health under Grant R01HL073647 and Grant R01HL087797.

\section{References}

1. Robinson G, Davies J. Continuum robots-A state of the art. Proc IEEE Int Conf Robot Autom. 1999; 4:2849-2854.

2. Camarillo D, Milne C, Carlson C, Salisbury KJ. Mechanics modeling of tendon-driven continuum manipulators. IEEE Trans Robot. Dec; 2008 24(6):1262-1273.

3. Jones B, Walker I. Kinematics for multi-section continuum robots. IEEE Trans Robot. Feb; 2006 22(1):45-53.

4. Simaan, N.; Taylor, R.; Flint, P. A dexterous system for laryngeal surgery. Proc. IEEE Int. Conf. Robot. Autom; New Orleans, LA. Apr. 2004; p. 351-357.

5. Webster R, Romano J, Cowan N. Mechanics of precurved-tube continuum robots. IEEE Trans Robot. Feb; 2009 25(1):67-78.

6. Dupont P, Lock J, Itkowitz B, Butler E. Design and control of concentric tube robots. IEEE Trans Robot. Apr; 2010 26(2):209-225. [PubMed: 21258648]

7. Sears, P.; Dupont, P. A steerable needle technology using curved concentric tubes. Proc. IEEE/RSJ Int. Conf. Intell. Robots Syst; Beijing, China. 2006. p. 2850-2856. 
8. [Online]. Available: http://www.hansenmedical.com/.

9. Webster RJ, Kim JS, Cowan NJ, Chirikjian GS, Okamura AM. Nonholonomic modeling of needle steering. Int J Robot Res. 2006; 25(5-6):509-525.

10. Hogan N. Impedance control: An approach to manipulation: Part I, II, III. J Dyn Syst Meas Control. 1985; 107(1):1-24.

11. Salisbury, JK. Active stiffness control of a manipulator in cartesian coordinates. Proc. IEEE Conf. Decis. Control; 1980. p. 95-100.

12. Mahvash M, Okamura A. Friction compensation for enhancing transparency of a teleoperator with compliant transmission. IEEE Trans Robot Autom. Dec; 2007 23(6):1240-1246.

13. Hashtrudi-Zaad K, Salcudean SE. Transparency in time-delayed systems and the effect of local force feedback for transparent teleoperation. IEEE Trans Robot Autom. Feb; 2002 18(1):108-114.

14. Xu K, Simaan N. An investigation of the intrinsic force sensing capabilities of continuum robots. IEEE Trans Robot. Jun; 2008 24(3):576-587.

15. Pai D. Strands: Interactive simulation of thin solids using cosserat models. Proc Eurograph. 2002; 21:347-352.

16. Antman, SS. Nonlinear Problems of Elasticity. New York: Springer-Verlag; 1995.

17. Lock, J.; Laing, G.; Mahvash, M.; Dupont, P. Quasistatic modeling of concentric tube robots with external loads. Proc. IEEE/RSJ Int. Conf. Intell. Robots Syst; Taipei, Taiwan. May 2010; p. 2325-2332.

18. Camarillo, DB.; Loewke, K.; Carlson, C.; Salisbury, KJ. Vision based 3-D shape sensing of flexible manipulators. Proc. IEEE Int. Conf. Robot. Autom; Pasadena, CA. 2008. p. 2940-2947.

19. Xu K, Simaan N. Analytic formulation for kinematics, statics and shape restoration of multibackbone continuum robots via elliptic integrals. ASME J Mech Robot. 2010; 2(1):1-13.

20. Chiou C, Shahinpoor M. Dynamic stability analysis of a twolink force-controlled flexible manipulator. ASME J Dyn Sys Meas. 1990; 112(2):661-666.

21. Ott C, Albu-Schaffer A, Hirzinger G. On the passivity-based impedance control of flexible joint robots. IEEE Trans Robot. Apr; 2008 24(2):416-429.

22. Murray, M.; Li, Z.; Sastry, SS. A Mathematical Introduction to Robotic Manipulation. 1. Boca Raton, FL: CRC; 1994.

23. Trivedi D, Lotfi A, Rahn C. Geometrically exact models for soft robotic manipulators. IEEE Trans Robot. Aug; 2008 24(4):773-780.

24. Bishop R. There is more than one way to frame a curve. Amer Math Monthly. 1975; 82(3):246251.

25. Crouch PE, Grossman R. Numerical integration of ordinary differential equations on manifolds. J Nonlinear Sci. 1993; 3(1):1-33.

26. Park, J.; Chung, W.; Youm, Y. Geometric numerical integration algorithms for articulated multibody systems. Proc. IEEE/RSJ Int. Conf. Intell. Robots Syst; 2004. p. 2803-3808.

27. Atkinson, K. An Introduction to Numerical Analysis. 2. New York: Wiley; 1989.

28. Mahvash, M.; Gwilliam, J.; Agarwal, R.; Vagvolgyi, B.; Su, L.; Yuh, D.; Okamura, A. Force feedback surgical teleoperator: controller design and palpation experiments. Proc. Symp. Haptic Interfaces, Virtual Environ. Teleoperator Syst; Reno, NV. Mar. 2008; p. 465-471.

\section{Biographies}

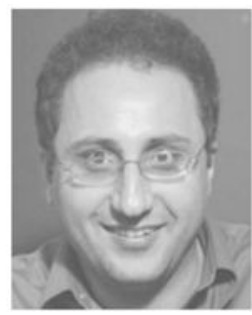

IEEE Trans Robot. Author manuscript; available in PMC 2013 November 22. 
Mohsen Mahvash (M'05) received the Ph.D. degree in electrical engineering from McGill University, Montreal, QC, Canada, in 2002.

For two years, he was a Postdoctoral Fellow with Johns Hopkins University, Baltimore, MD, where he was also an Assistant Research Professor. Subsequently, he was an Assistant Research Professor with the Department of Mechanical Engineering, Boston University,

Boston, MA. He is currently with Harvard Medical School (Brigham and Women's Hospital and Cardiac Surgery Department, Veterans Affairs Boston Healthcare System), West Roxbury, MA. His research interests include medical robotics, physics-based modeling, haptics, and control.

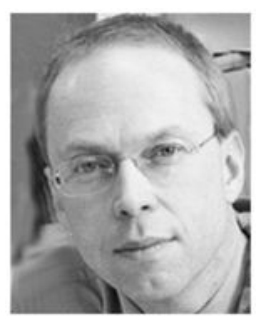

Pierre E. Dupont (M'99-SM'03-F'11) received the B.S., M.S., and Ph.D. degrees in mechanical engineering from Rensselaer Polytechnic Institute, Troy, NY, in 1982, 1984, and 1988 , respectively.

From 1988 to 1990, he was a Postdoctoral Fellow with the School of Engineering and Applied Sciences, Harvard University, Cambridge, MA. He was a Professor of mechanical engineering and biomedical engineering with Boston University, Boston, MA. He is currently the Chief of pediatric cardiac bioengineering with Children's Hospital Boston, Harvard Medical School, Boston, where he is engaged in developing instrumentation and imaging technology for minimally invasive surgery. 

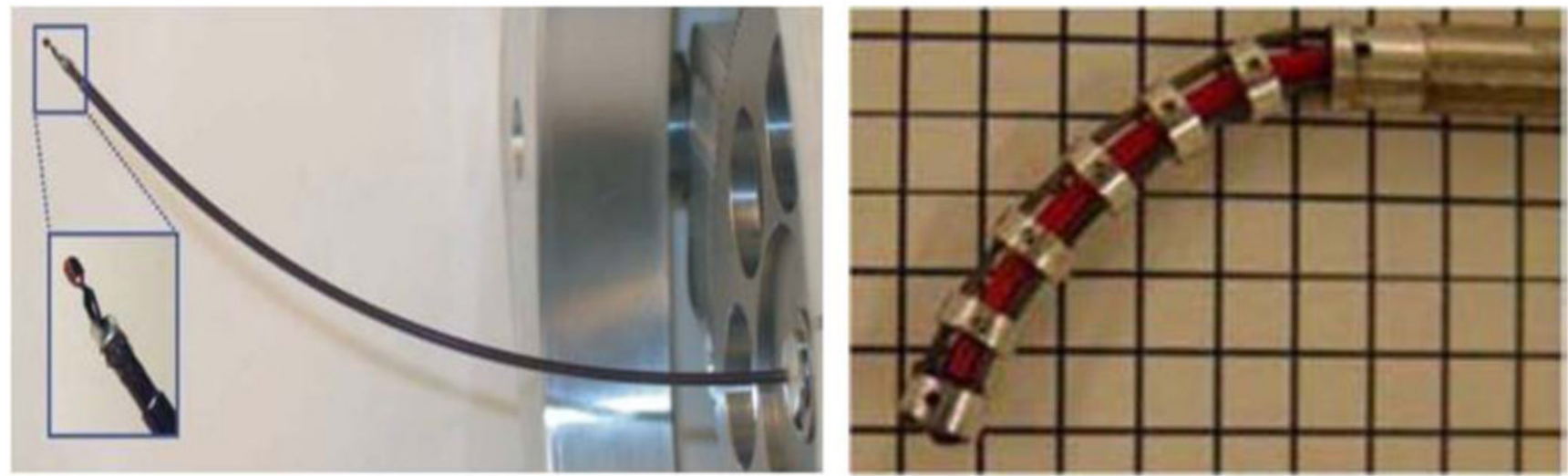

Fig. 1.

Examples of continuum manipulators. (a) Twenty-centimeter-long concentric tube robot with 1-mm-wide tip-mounted forceps. (b) Four-millimeter diameter multi-backbone robot [4]. 


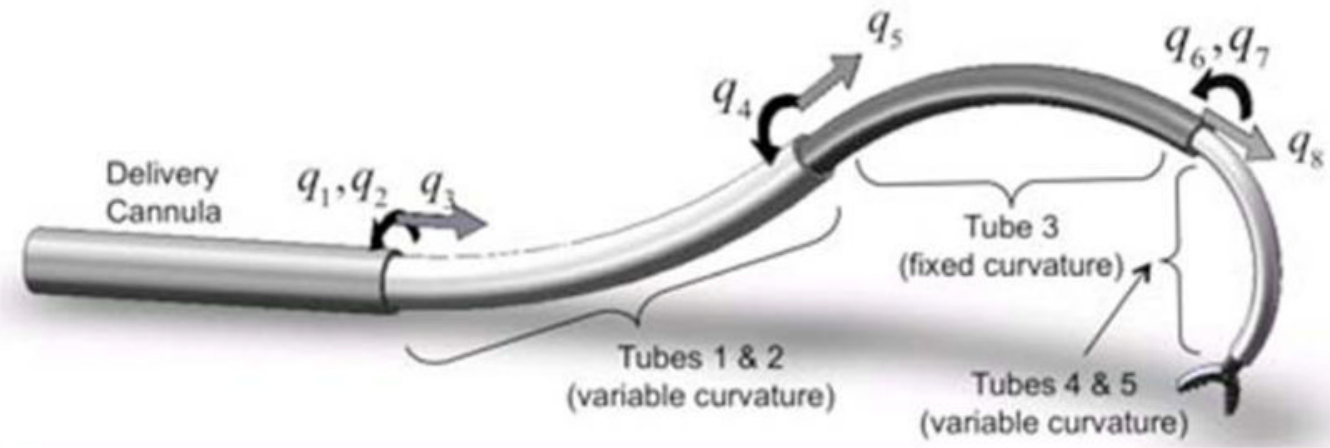

(a)

(variable curvature)

(b)

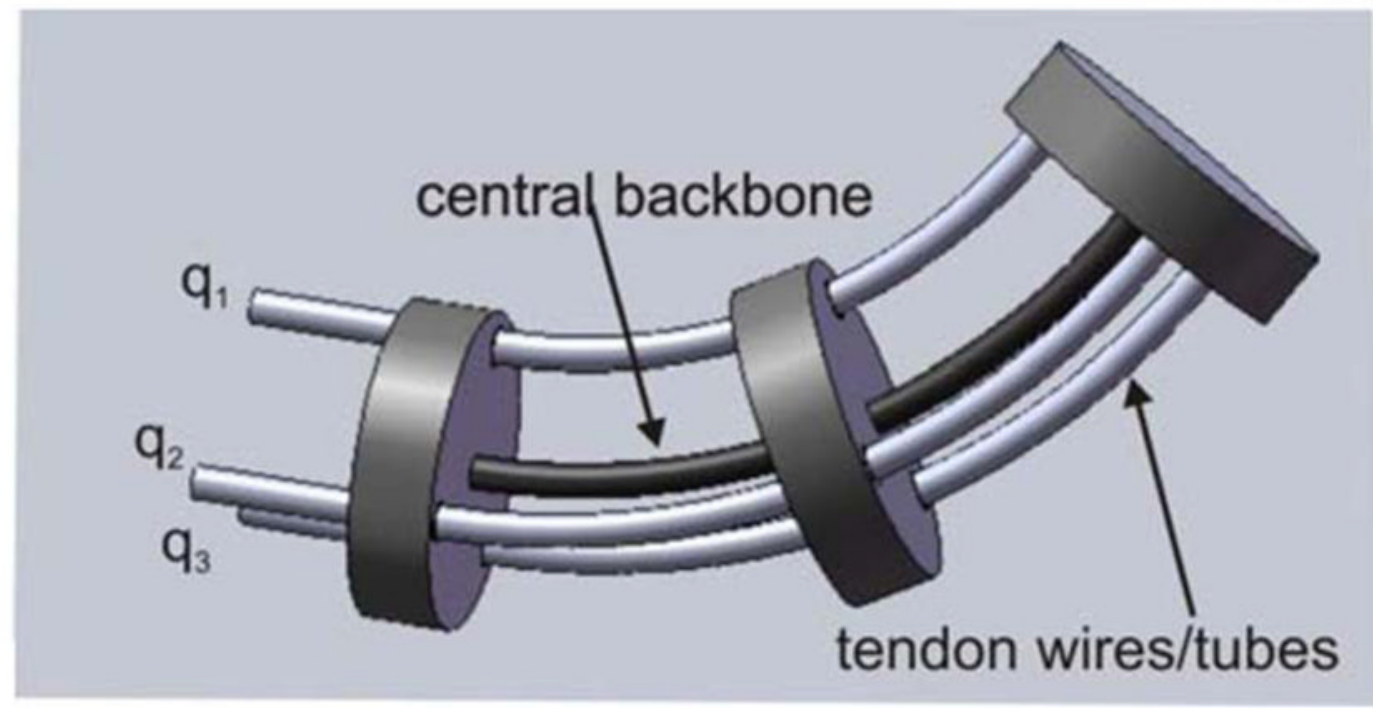

Fig. 2.

Schematics of two types of continuum robots. (a) Concentric tube robot. (b) Tendon-driven robot with flexible backbone. 


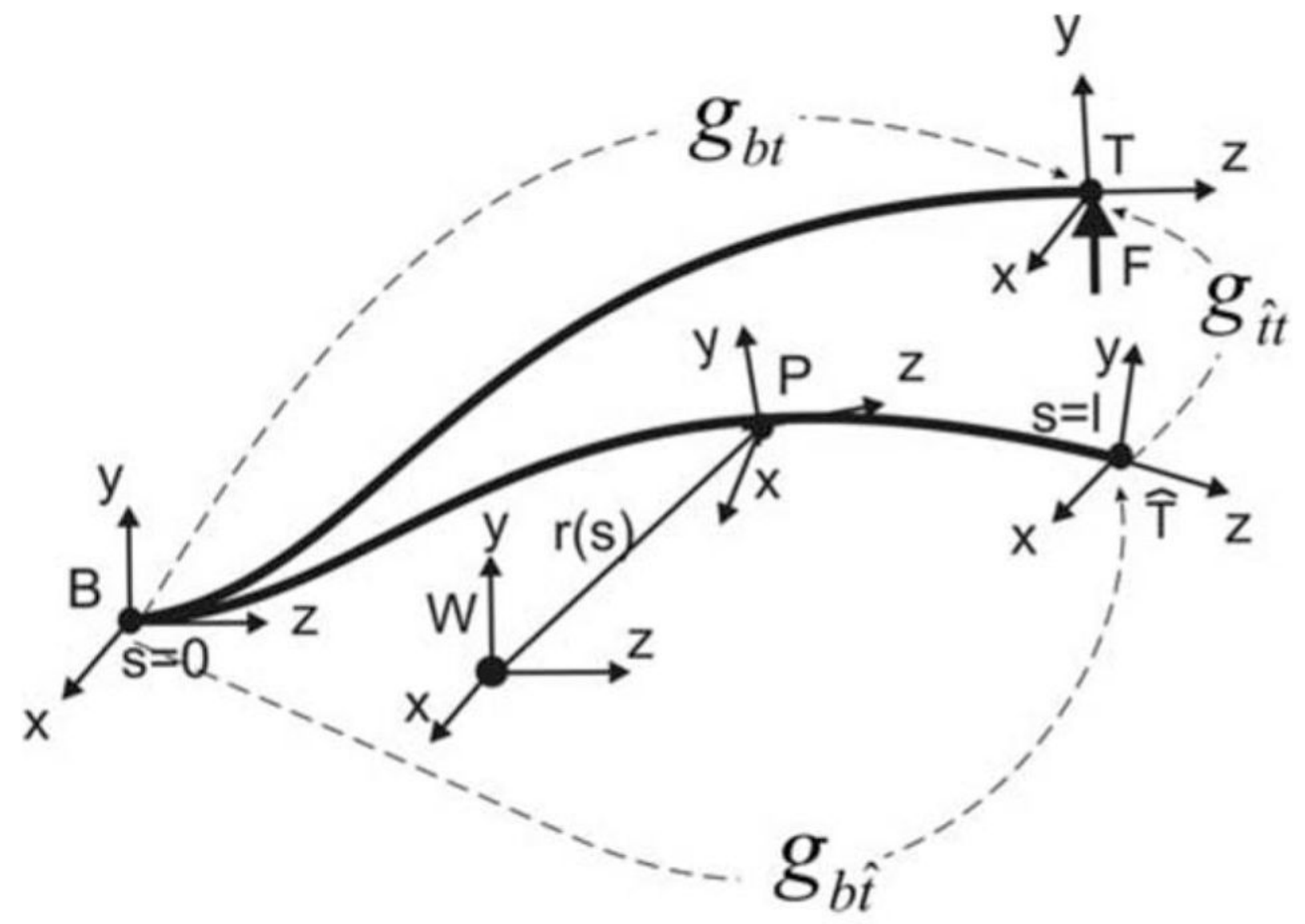

Fig. 3.

Continuum robot represented as a space curve (solid line). $W$ is world coordinate frame, while $B, P, T$, and $T$ are robot body frames. $T$ and $T$ are tip frames without and with the application of tip wrench $F$, respectively. Coordinate transformations are denoted by $g_{b t}, g_{b t}$. and $g_{t t}$ 


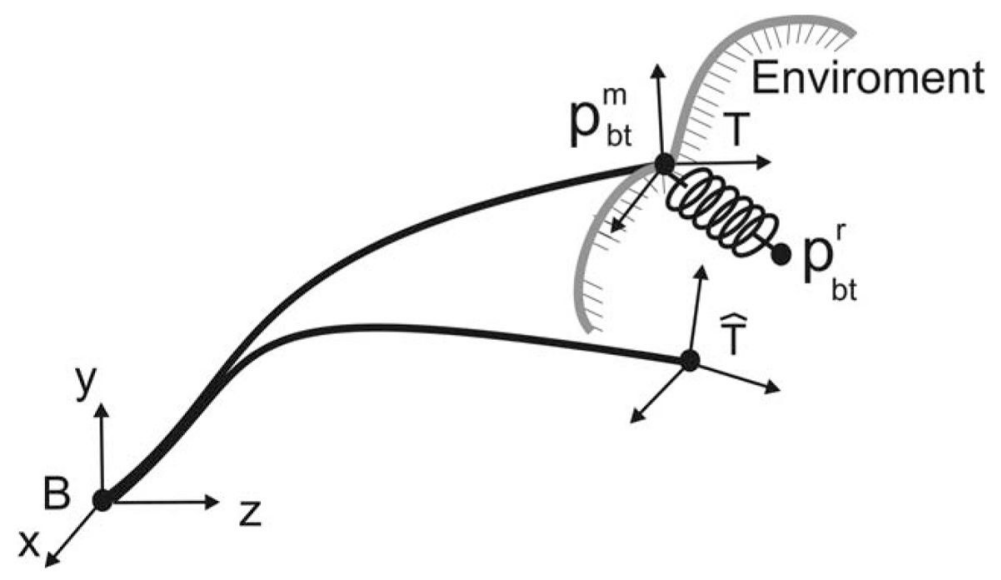

Fig. 4.

Robot in contact with environment. The stiffness controller implements a virtual linear spring at the robot's tip. Desired actuator positions are such that when robot is deflected from unloaded tip configuration $T$ to configuration $T$, the desired tip spring force is generated, and the desired tip orientation is achieved. 


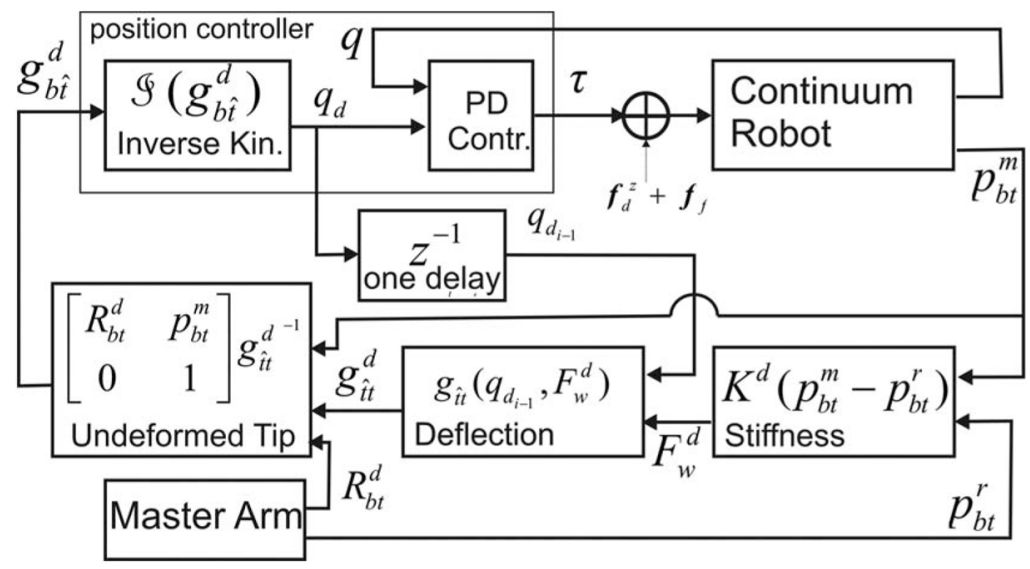

Fig. 5.

Block diagram of the stiffness controller. A single fixed-point iteration is performed per controller cycle. 


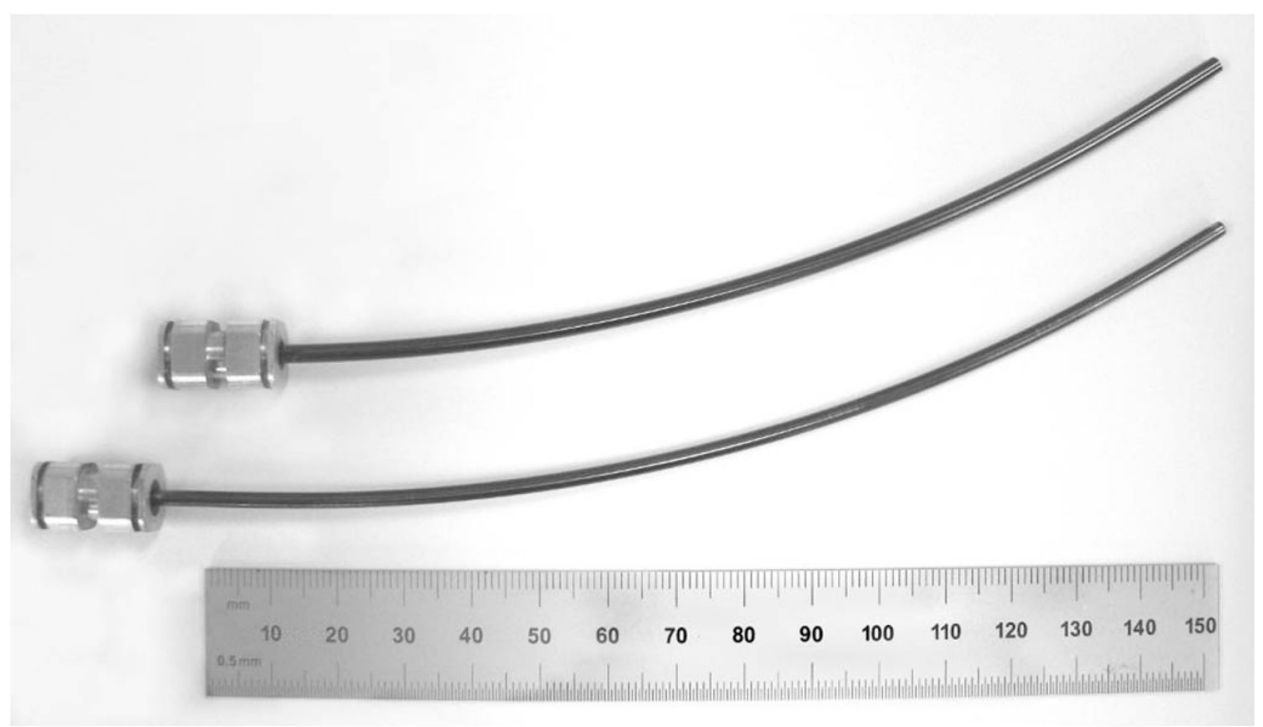

Fig. 6.

Robot component tubes. NiTi tubes assemble concentrically to form a 3-DOF robot. Tubes are of equal initial curvature and bending stiffness. The outer tube (top) has an outer diameter of $2.8 \mathrm{~mm}$ and inner diameter of $2.5 \mathrm{~mm}$. The inner tube (bottom) has an outer diameter of $2.4 \mathrm{~mm}$ and an inner diameter of $2.0 \mathrm{~mm}$. 


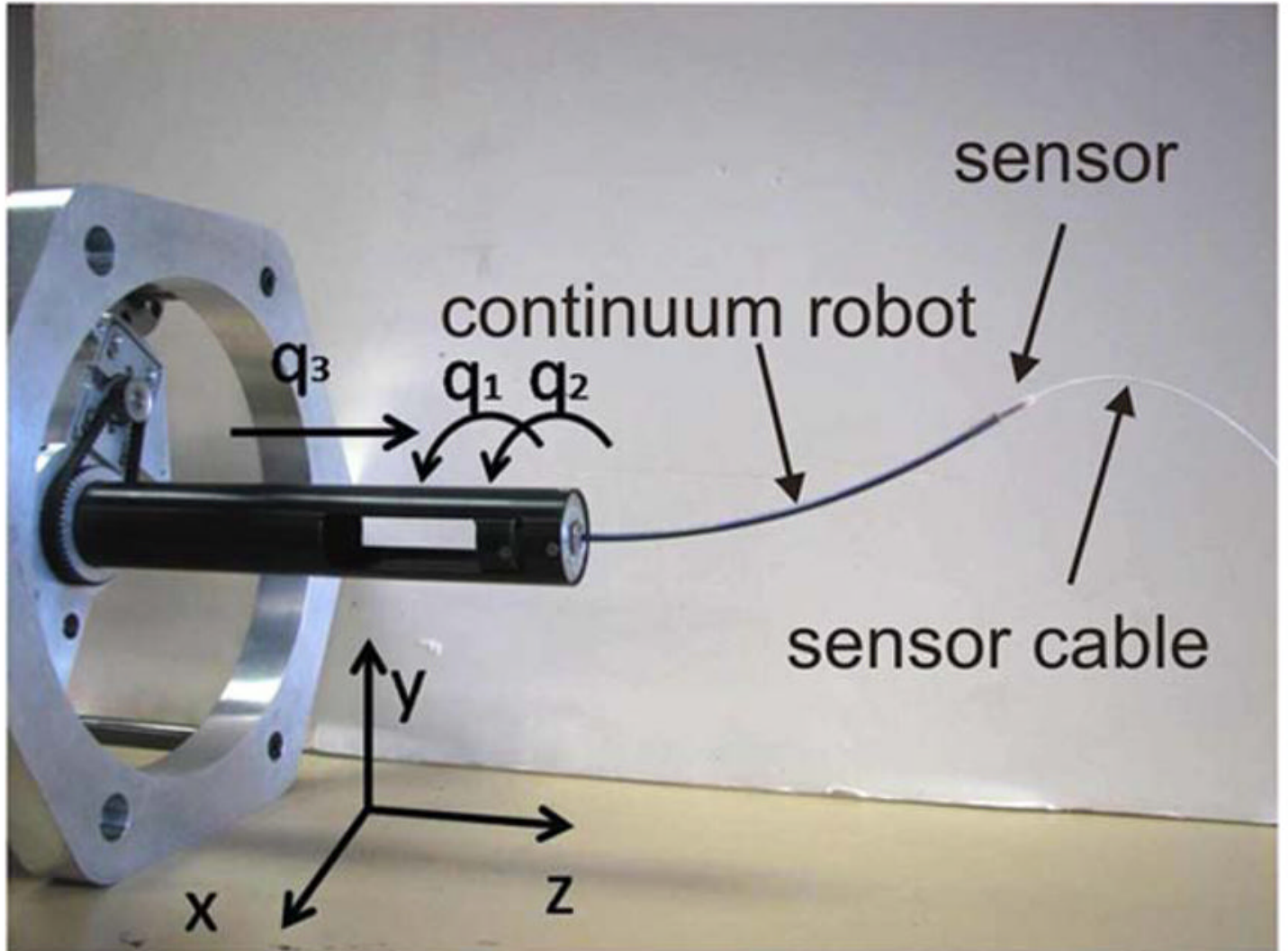

Fig. 7.

Concentric tube manipulator mounted in its drive system. Electromagnetic sensor is shown attached to robot tip. Actuator variables $q_{1}$ and $q_{2}$ control rotation of two tubes and $q_{3}$ controls translation of tube pair. Curvature is varied by relative rotation of the tubes. 


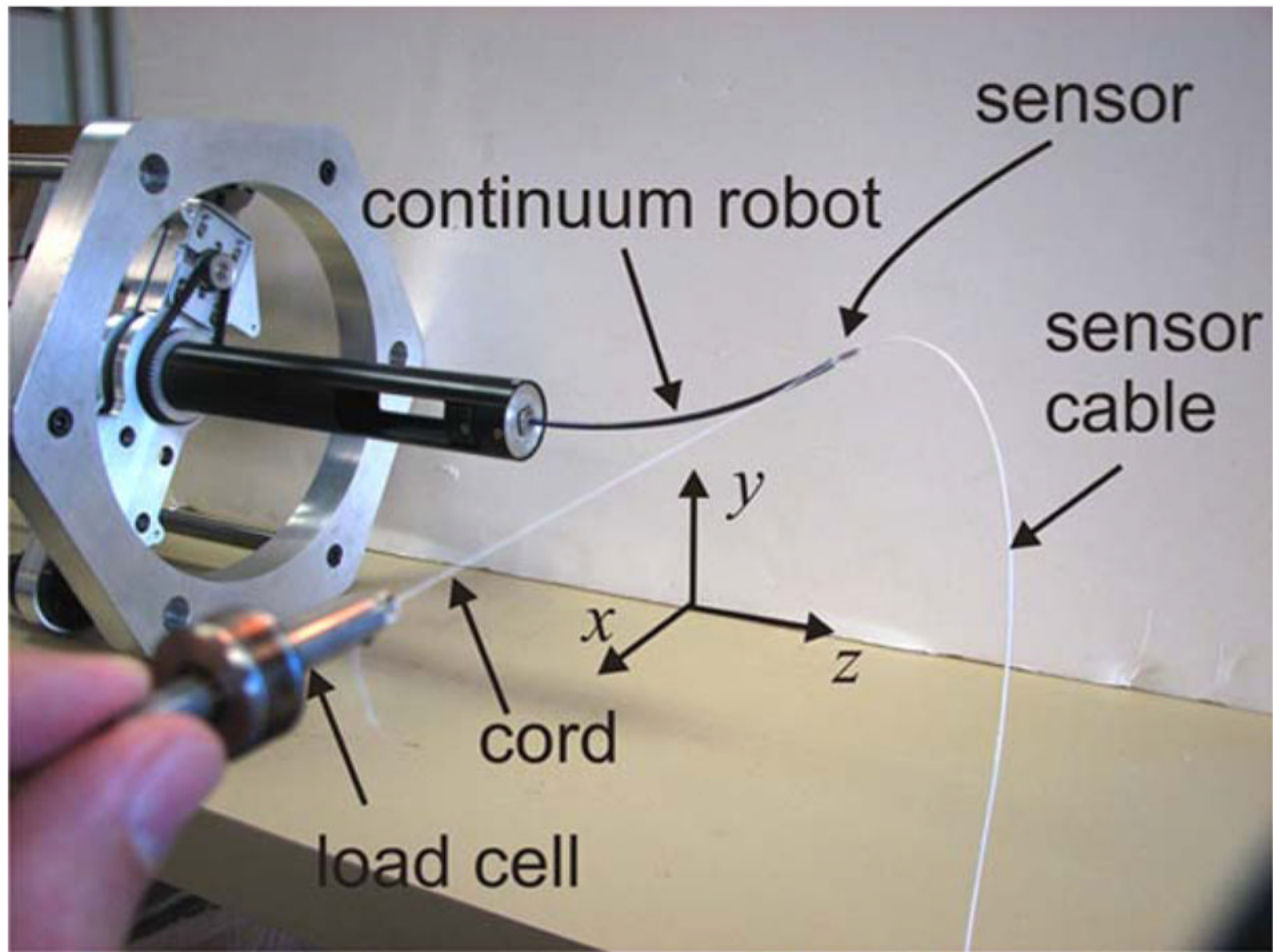

Fig. 8.

Interaction with a moving environment. Environment motion is produced by manual loading of the robot tip through a cord attached to a load cell. 


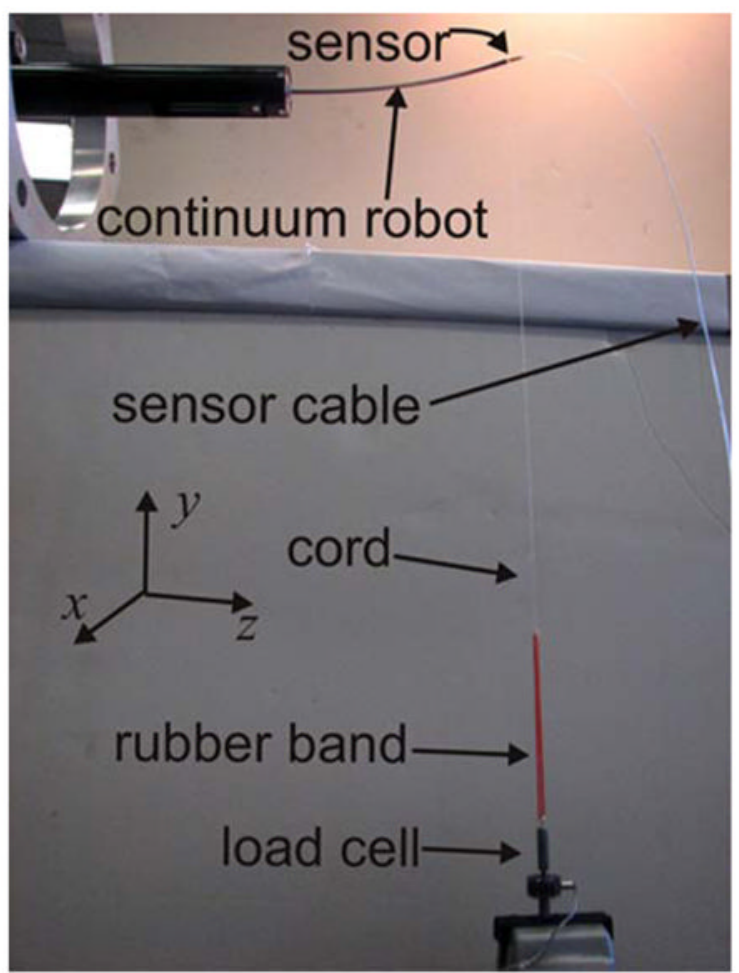

Fig. 9.

Interaction with a fixed elastic environment. Environment is modeled by a rubber band connecting a tip-mounted cord and a fixed load cell. 


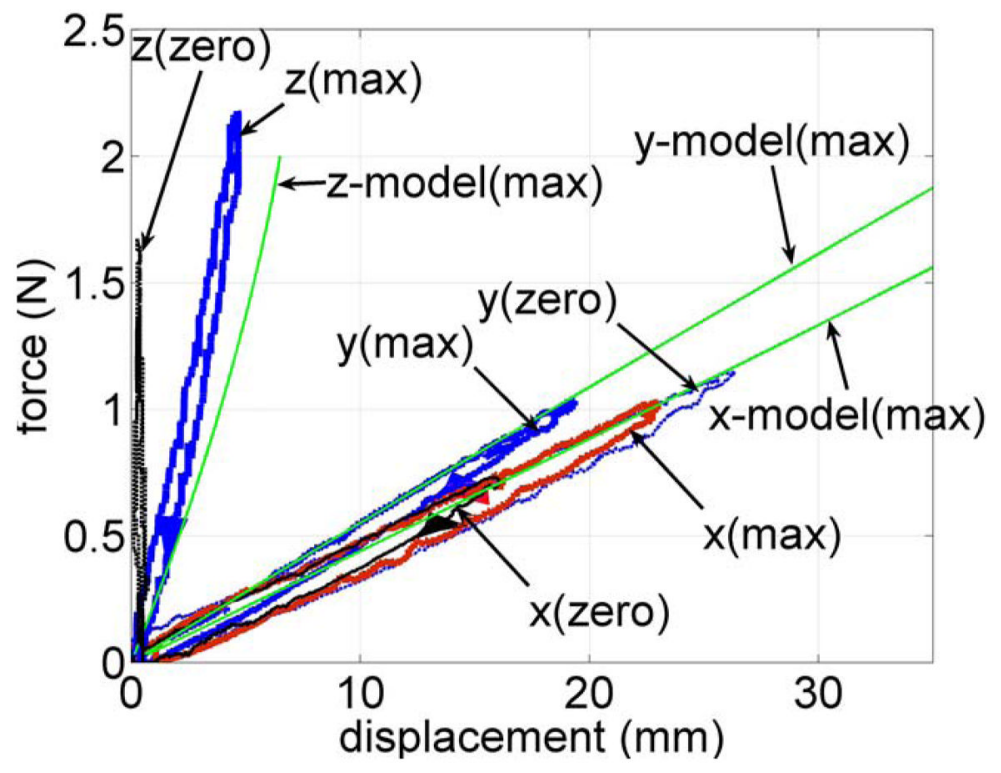

Fig. 10.

Force versus displacement of the robot tip in the world coordinate directions $\{x, y, z\}$ shown in Fig. 7. Both experimental data and predictions from the calibrated deflection model are shown for the maximum curvature configuration labeled (max) and zero curvature configuration labeled (zero) of Fig. 7. 


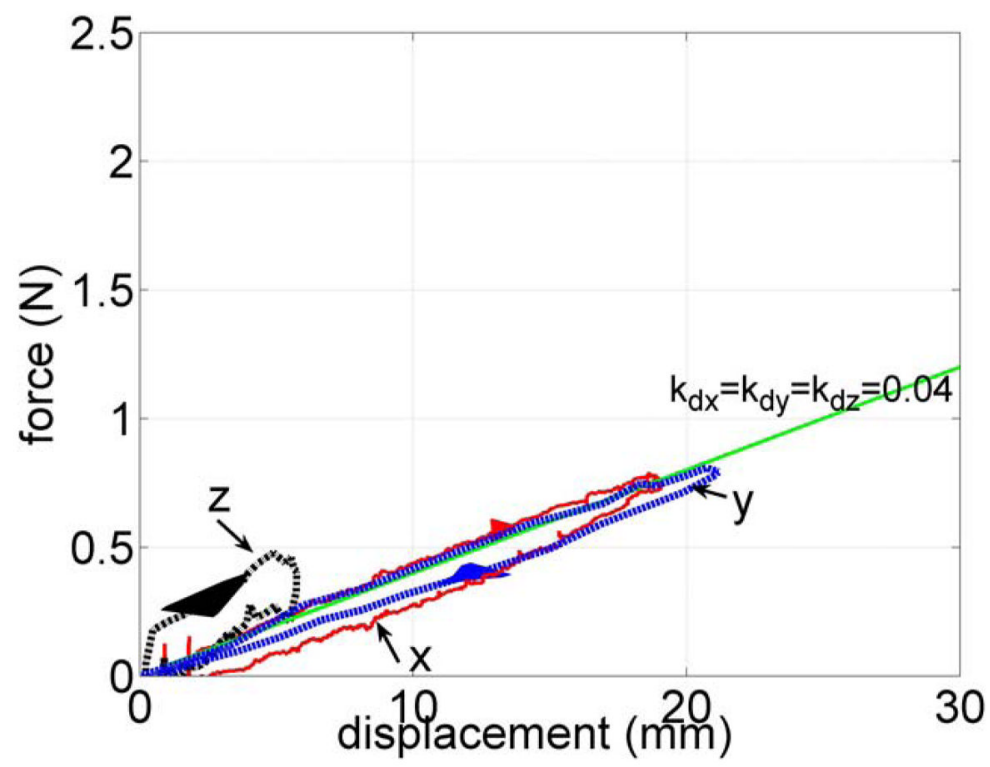

Fig. 11.

Tip force versus displacement in the three coordinate directions for a noncontact robot curvature of $u^{W}=[(1 / 320 \mathrm{~mm}) 00]^{T}$ and desired tip stiffness of $K_{d}=\operatorname{diag}(0.040 .040 .04)$ $\mathrm{N} / \mathrm{mm}$. 


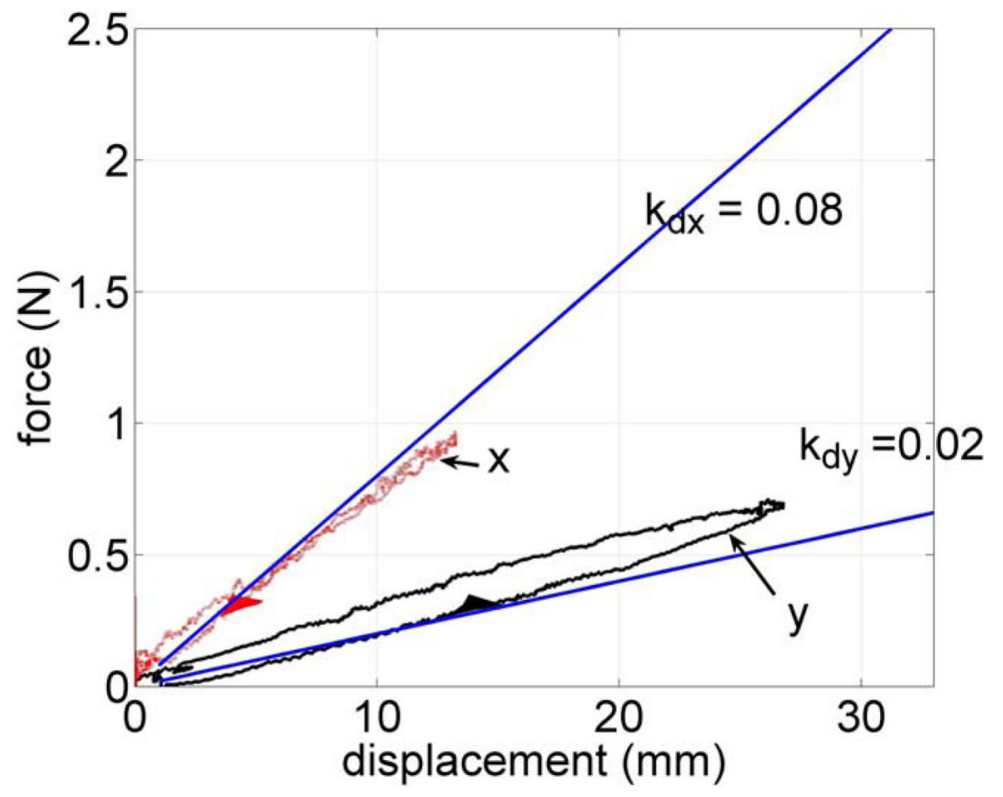

Fig. 12.

Tip force versus displacement in the $x$ - and $y$-coordinate directions for a noncontact robot curvature of $u^{W}=\left[\begin{array}{lll}0 & 0 & 0\end{array}\right]^{T}$ and desired tip stiffness of $K_{d}=\operatorname{diag}\left(\begin{array}{llll}0.02 & 0.08 & 0.2\end{array}\right) \mathrm{N} / \mathrm{mm}$. 


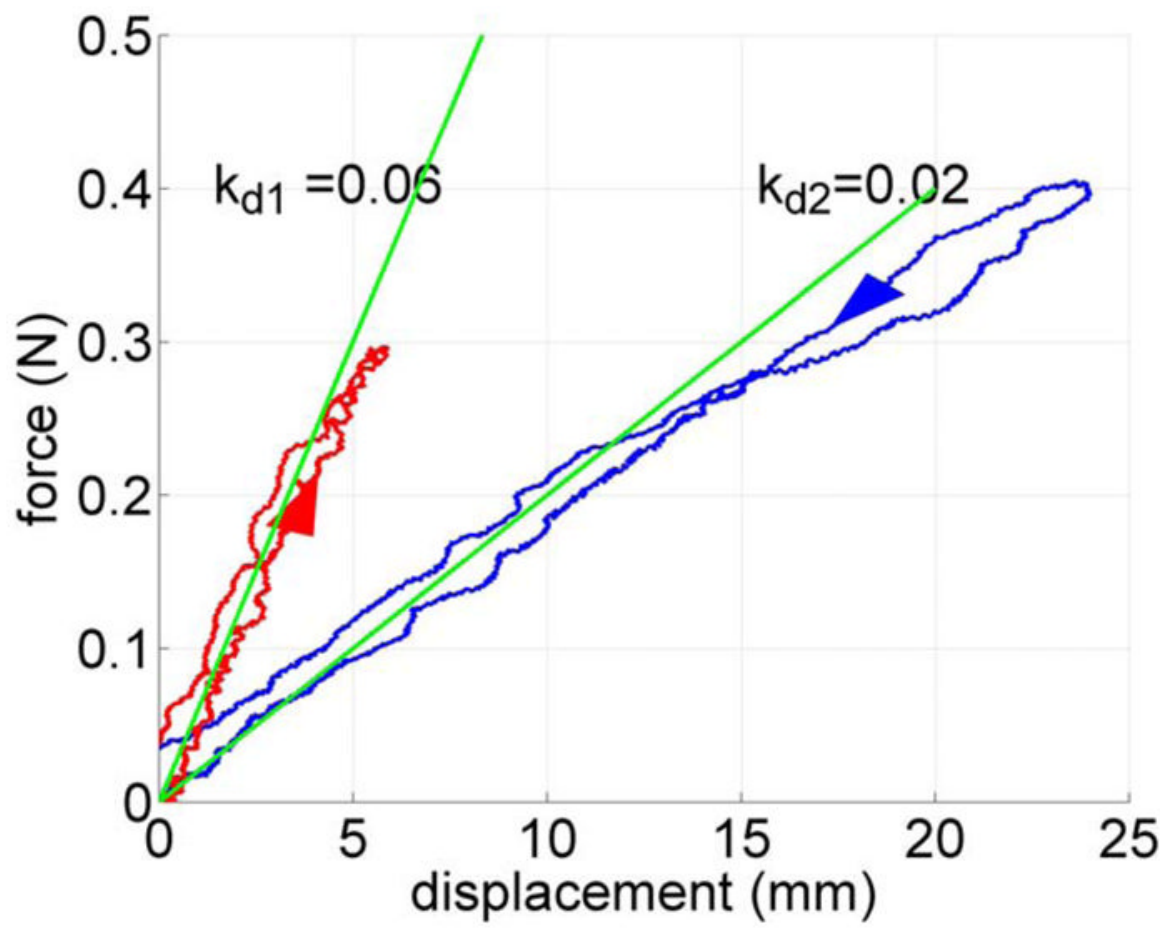

Fig. 13.

Tip force versus displacement in the $y$-direction during contact with a soft stationary environment for desired stiffness values of 0.02 and $0.06 \mathrm{~N} / \mathrm{mm}$. 


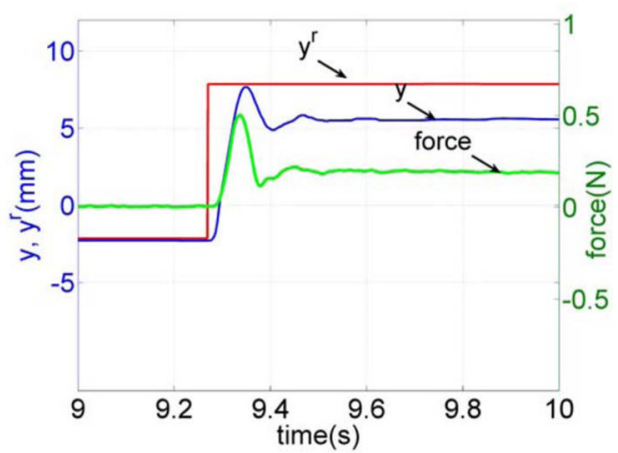

(a)

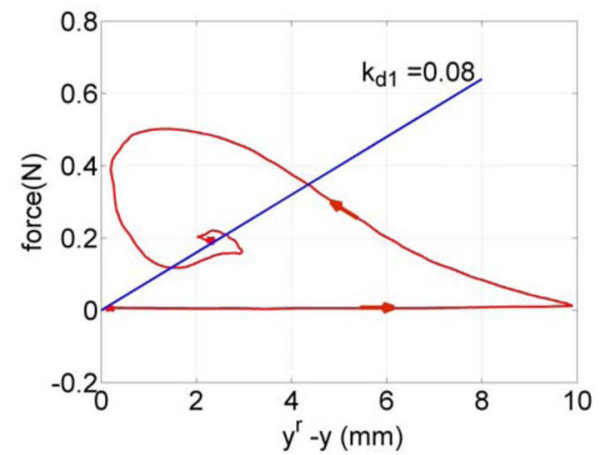

(b)

Fig. 14.

Stiffness controller step response. Step change in reference position $y^{r}$ is applied such robot transitions from free space to contact with environment through cord. Desired stiffness is $k_{d}$ $=0.08 \mathrm{~N} / \mathrm{mm}$. (a) Time response plot of $y^{r}, y$, and $f_{y}$. (b) Tip force versus virtual spring displacement $y r-y$. 


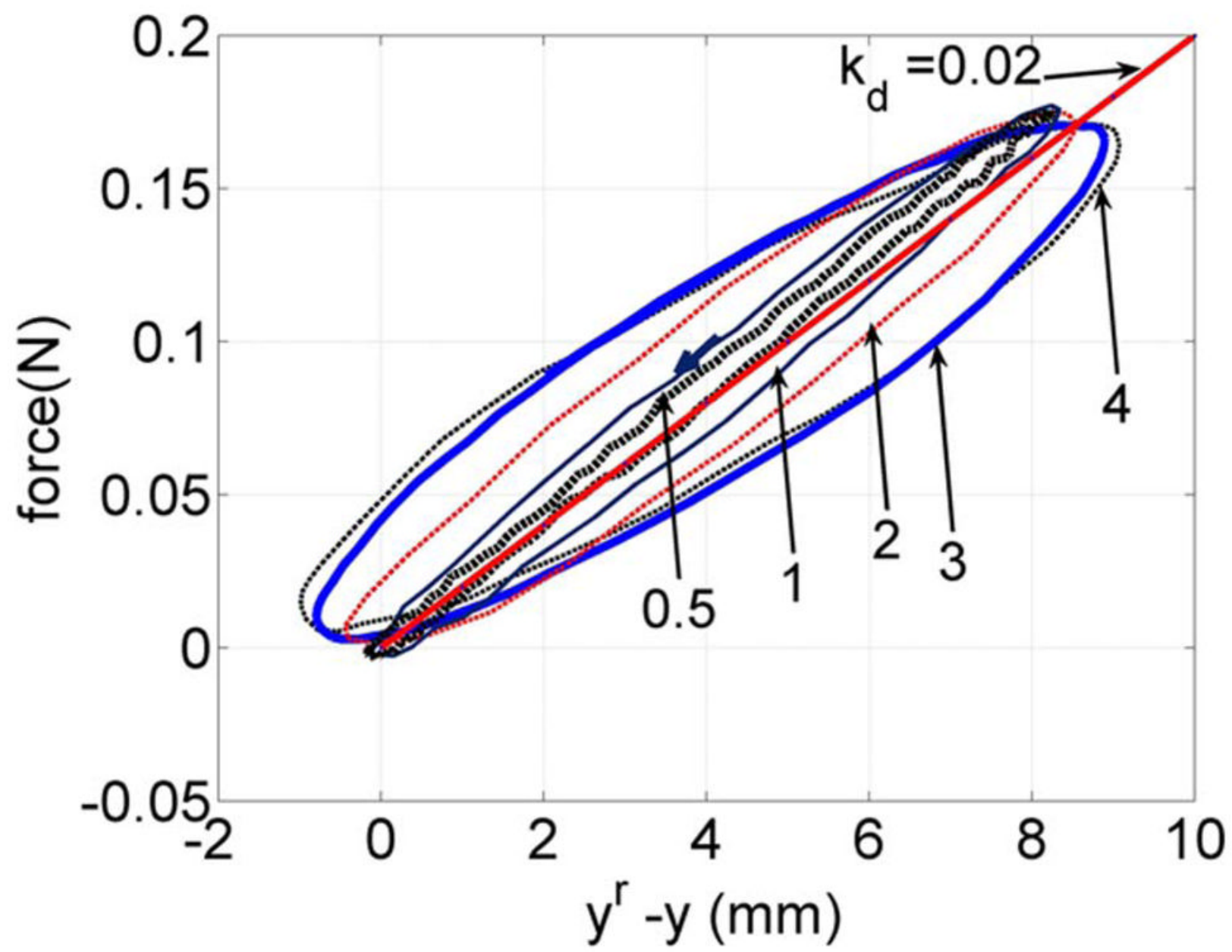

Fig. 15.

Force versus virtual spring displacement for sinusoidal inputs of $y^{r}$. Responses are shown for an input amplitude of $12 \mathrm{~mm}$ and frequencies of $0.5,1,2,3$, and $4 \mathrm{~Hz}$. Desired stiffness is $k_{d}=0.02 \mathrm{~N} / \mathrm{mm}$. 
TABLE 1

\section{Nomenclature}

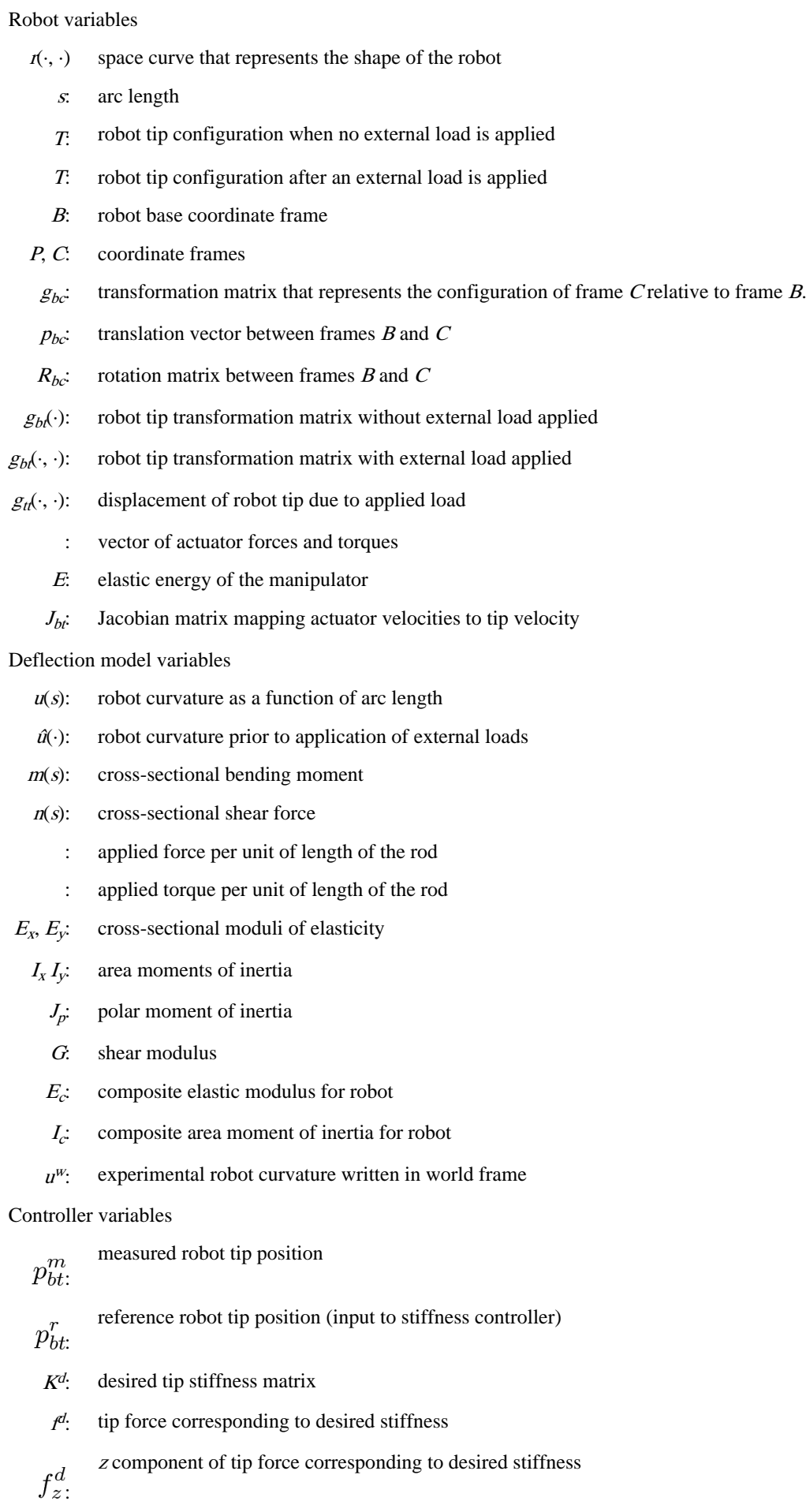




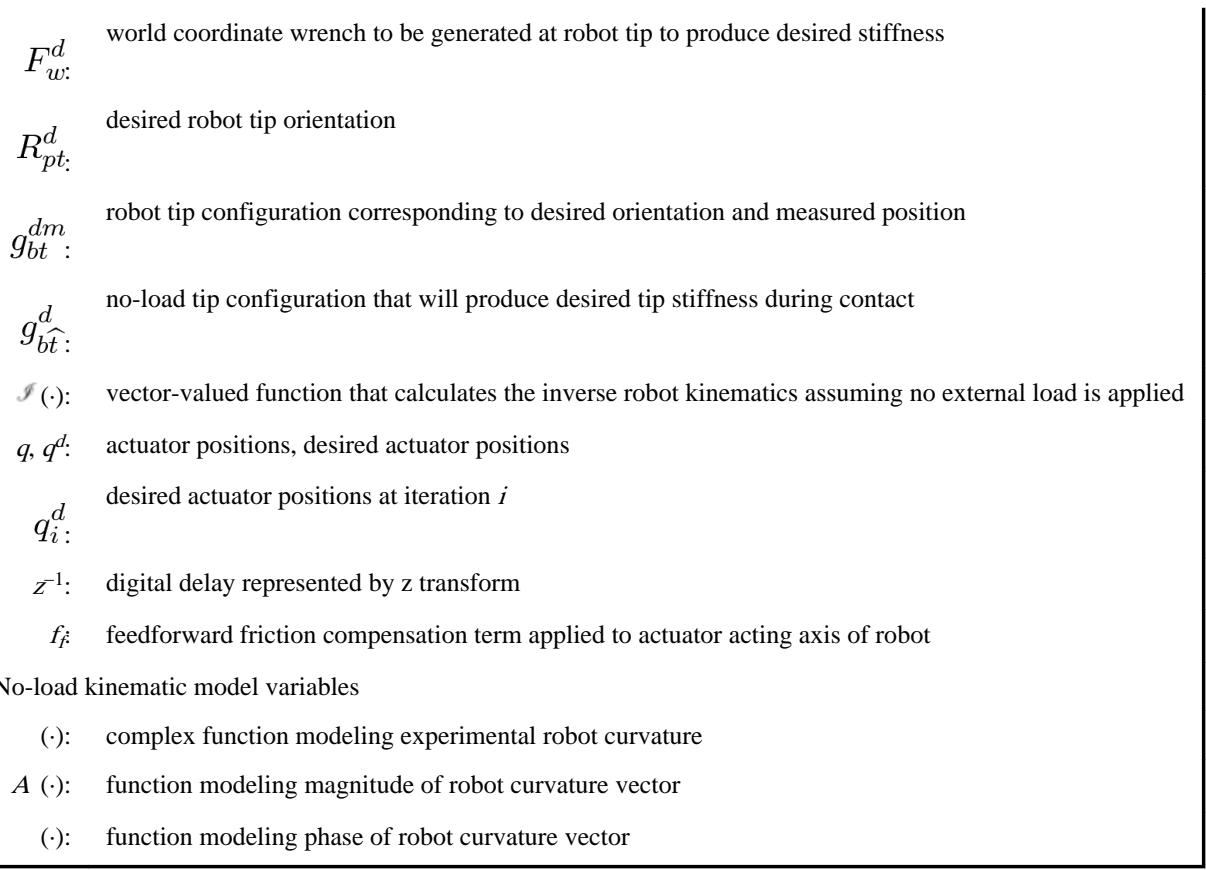

